\title{
ON THE IDEAL CLASS GROUPS OF IMAGINARY ABELIAN FIELDS WITH SMALL CONDUCTOR
}

\author{
KUNIAKI HORIE AND HIROKO OGURA
}

\begin{abstract}
Let $k$ be any imaginary abelian field with conductor not exceeding 100 , where an abelian field means a finite abelian extension over the rational field $\mathbf{Q}$ contained in the complex field. Let $C(k)$ denote the ideal class group of $k, C^{-}(k)$ the kernel of the norm map from $C(k)$ to the ideal class group of the maximal real subfield of $k$, and $f(k)$ the conductor of $k ; f(k) \leq 100$. Proving a preliminary result on 2-ranks of ideal class groups of certain imaginary abelian fields, this paper determines the structure of the abelian group $C^{-}(k)$ and, under the condition that either $[k: \mathbf{Q}] \leq 23$ or $f(k)$ is not a prime $\geq 71$, determines the structure of $C(k)$.
\end{abstract}

We shall mean by an abelian field a finite abelian extension over the rational field $\mathbf{Q}$ contained in the complex field $\mathbf{C}$. For any abelian field $k$, let $h(k)$ denote the class number of $k, C(k)$ the ideal class group of $k, r_{2}(k)$ the 2-rank of $C(k), k^{+}$the maximal real subfield of $k, \operatorname{Cir}(k)$ the (group of) circular units of $k$ in the sense of Sinnott [14], $\operatorname{Cir}^{+}(k)$ the group of real elements of $\operatorname{Cir}(k)$, and $\operatorname{Cir}^{*}(k)$ the group of totally positive elements of $k^{+}$ in $\operatorname{Cir}(k) ; \operatorname{Cir}^{*}(k) \subseteq \operatorname{Cir}^{+}(k)=\operatorname{Cir}(k) \cap k^{+}$. Furthermore, for each subfield $k^{\prime}$ of $k$, let $N_{k / k^{\prime}}$ denote the norm map from $k$ to $k^{\prime}$. Given any positive integer $m$ not congruent to 2 modulo 4 , let $\mathbf{K}_{m}$ denote the cyclotomic field of $m$-th roots of unity in $\mathbf{C}$, namely, let

$$
\mathbf{K}_{m}=\mathbf{Q}\left(\zeta_{m}\right), \quad \text { where } \zeta_{m}=e^{2 \pi i / m} .
$$

In this paper, we shall first prove the following:

I. Let $p$ and $q$ be distinct prime numbers. Let $K$ be an imaginary abelian field with conductor a p-power, $L$ an imaginary abelian field with conductor $a$ $q$-power, and $t$ the number of distinct prime ideals dividing $p$ of the composite $K^{+} L^{+}$. Let $\delta=0$ or 1 according as the degree $\left[K^{+}: \mathbf{Q}\right]$ is odd or even. Assume further that $h\left(K^{+} L^{+}\right)$is odd and that only a prime ideal of $K L$ divides $q$. Then

$$
\begin{aligned}
& r_{2}\left(K L^{+}\right) \geq r_{2}\left(K^{+} L\right)+t-\delta-1 \quad \text { if } \operatorname{Cir}^{*}\left(K^{+} L\right) \subseteq N_{K L^{+} / K^{+} L^{+}}\left(K L^{+}\right) ; \\
& r_{2}\left(K L^{+}\right) \leq r_{2}\left(K^{+} L\right)+t-2 \quad \text { unless } \operatorname{Cir}^{*}\left(K^{+} L\right) \subseteq N_{K L^{+} / K^{+} L^{+}}\left(K L^{+}\right) .
\end{aligned}
$$

Received by the editors September 8, 1994.

1991 Mathematics Subject Classification. Primary 11R20, 11R29; Secondary 11R16, 11R18, 11 R27.

Supported in part by Grant-in-Aid for Scientific Research (No. 06640080), Ministry of Education, Science, and Culture, Japan. 
Our proof of this assertion I is mainly based upon an algebraic interpretation by Sinnott [14] of the analytic class number formula. Verifying

$$
\operatorname{Cir}^{*}\left(\mathbf{K}_{13}(\sqrt{5})\right) \subseteq N_{\mathbf{K}_{5} \mathbf{K}_{13}^{+} / \mathbf{K}_{13}^{+}(\sqrt{5})}\left(\mathbf{K}_{5} \mathbf{K}_{13}^{+}\right)
$$

and using some results in $[4,11,12]$, such as $h\left(\mathbf{K}_{13}^{+}(\sqrt{5})\right)=1$ and $h\left(\mathbf{K}_{5} \mathbf{K}_{13}^{+}\right)$ $=2^{4}$, we shall next see from I that $C\left(\mathbf{K}_{5} \mathbf{K}_{13}^{+}\right)$is an elementary abelian 2-group of rank 4 .

Now, for any abelian field $k$, let $f(k)$ denote the conductor of $k$. If $k$ is imaginary, let $h^{-}(k)$ denote the relative class number of $k$ and $C^{-}(k)$ the kernel of the norm map $C(k) \rightarrow C\left(k^{+}\right)$. We then call $C^{-}(k)$ the relative class group of $k$. Note here that since the norm map $C(k) \rightarrow C\left(k^{+}\right)$is surjective by class field theory, the condition $h\left(k^{+}\right)=1$ is equivalent to the condition $C(k)=C^{-}(k)$ and the relative class number $h^{-}(k)$ equals the order of the relative class group $C^{-}(k) ; h^{-}(k)=\left|C^{-}(k)\right|$. As usual, $\mathbf{Z}$ will denote the ring of (rational) integers and $\mathbf{N}$ the set of positive integers. The additive group of $\mathbf{Z}$ will be denoted by the same letter $\mathbf{Z}$. We mean by a cyclic field an abelian field whose Galois group over $\mathbf{Q}$ is cyclic. For each odd prime $l$ and each $n \in \mathbf{N}$ dividing $l-1$, let $\mathbf{K}_{l, n}$ denote the subfield of $\mathbf{K}_{l}$ with degree $n$, so that $\mathbf{K}_{l, n}$ is a cyclic field. In addition, let $\mathbf{K}_{60,4}$ denote the unique imaginary cyclic field of degree 4 with conductor 60 :

$$
\mathbf{K}_{60,4}=\mathbf{Q}\left(\left(\zeta_{5}-\zeta_{5}^{-1}\right) \sqrt{3}\right) .
$$

Put

$$
\mathbf{L}_{65,12}=\mathbf{Q}\left(\left(\zeta_{5}-\zeta_{5}^{-1}\right)\left(\zeta_{13}-\zeta_{13}^{-1}\right)\left(\zeta_{13}^{5}-\zeta_{13}^{-5}\right)\right), \quad \mathbf{L}_{65,12}^{\prime}=\mathbf{Q}\left(\left(\zeta_{13}-\zeta_{13}^{-1}\right) \sqrt{5}\right) .
$$

These are the distinct imaginary cyclic fields of degree 12 with conductor 65 other than $\mathbf{K}_{5} \mathbf{K}_{13,3}$. Let $\mathbf{K}_{68,8}, \mathbf{K}_{68,4}$, and $\mathbf{K}_{96,8}$ denote the imaginary cyclic fields such that $f\left(\mathbf{K}_{68,8}\right)=f\left(\mathbf{K}_{68,4}\right)=68, f\left(\mathbf{K}_{96,8}\right)=96,\left[\mathbf{K}_{68,8}: \mathbf{Q}\right]=$ $\left[\mathbf{K}_{96,8}: \mathbf{Q}\right]=8$, and $\left[\mathbf{K}_{68,4}: \mathbf{Q}\right]=4$ :

$$
\begin{aligned}
& \mathbf{K}_{68,8}=\mathbf{Q}\left(\left(\zeta_{17}-\zeta_{17}^{-1}\right)\left(\zeta_{17}^{4}-\zeta_{17}^{-4}\right) \sqrt{-1}\right), \\
& \mathbf{K}_{68,4}=\mathbf{Q}(\sqrt{-17-4 \sqrt{17}}), \\
& \mathbf{K}_{96,8}=\mathbf{Q}\left(\left(\zeta_{32}+\zeta_{32}^{-1}\right) \sqrt{-3}\right) .
\end{aligned}
$$

Here, for each $z \in \mathbf{C}$ other than any non-negative real number, we understand that $\sqrt{z}$ denotes the square root of $z$ in $\mathbf{C}$ whose imaginary part is positive.

Let us write $\mathscr{H}$ for the family of imaginary abelian fields $k$ with $f(k) \leq$ 100. It is well known that the relative class numbers of all fields in $\mathscr{H}$ are calculated by Hasse [4]. We add in passing that $|\mathscr{H}|=369$. Now, once the structure of the abelian group $C^{-}\left(\mathbf{K}_{5} \mathbf{K}_{13}^{+}\right)=C\left(\mathbf{K}_{5} \mathbf{K}_{13}^{+}\right)$is determined, various results on abelian fields in $[1,2,3,4,5,6,7,8,9,10,11,12,15]$ help us to determine the structure, as abelian groups, of the relative class groups of all fields in $\mathscr{H}$.

II. The relative class group of an imaginary abelian field in $\mathscr{H}$ is not cyclic if and only if the imaginary abelian field coincides with one of the following 34 
fields:

$$
\begin{aligned}
& \mathbf{K}_{29}, \mathbf{K}_{41}, \mathbf{K}_{60,4}, \mathbf{K}_{65}, \mathbf{K}_{5} \mathbf{K}_{13}^{+}, \mathbf{K}_{5} \mathbf{K}_{13,3}, \mathbf{K}_{13}(\sqrt{5}), \mathbf{L}_{65,12}, \mathbf{L}_{65,12}^{\prime}, \\
& \mathbf{K}_{68,8}, \mathbf{K}_{68,4}, \mathbf{K}_{77}, \mathbf{K}_{7} \mathbf{K}_{11}^{+}, \mathbf{K}_{7}^{+} \mathbf{K}_{11}, \mathbf{K}_{7}^{+}(\sqrt{-21}), \mathbf{Q}(\sqrt{-21}), \mathbf{K}_{87}, \\
& \mathbf{K}_{29}^{+}(\sqrt{-3}), \mathbf{K}_{29,7}(\sqrt{-3}), \mathbf{K}_{29,7}(\sqrt{-87}), \mathbf{K}_{91}, \mathbf{K}_{7} \mathbf{K}_{13}^{+}, \mathbf{K}_{7} \mathbf{K}_{13,4}, \\
& \mathbf{K}_{7}(\sqrt{13}), \mathbf{K}_{7}^{+} \mathbf{K}_{13}, \mathbf{K}_{7}^{+} \mathbf{K}_{13,4}, \mathbf{K}_{7}^{+} \mathbf{K}_{13,3}(\sqrt{-91}), \mathbf{K}_{7}^{+}(\sqrt{-91}), \mathbf{K}_{96}, \\
& \mathbf{K}_{32}^{+}(\sqrt{-3}), \mathbf{K}_{96,8}(\sqrt{-1}), \mathbf{K}_{96,8}(\sqrt{3}), \mathbf{K}_{96,8}, \mathbf{K}_{99} .
\end{aligned}
$$

The maximal real subfield of each of these fields has class number 1 , and

$$
\begin{aligned}
& C\left(\mathbf{K}_{29}\right) \cong C\left(\mathbf{L}_{65,12}\right) \cong C\left(\mathbf{L}_{65,12}^{\prime}\right) \cong C\left(\mathbf{K}_{29,7}(\sqrt{-3})\right) \cong C\left(\mathbf{K}_{7}^{+}(\sqrt{-91})\right) \\
& \cong(\mathbf{Z} / 2 \mathbf{Z})^{3} \text {, } \\
& C\left(\mathbf{K}_{41}\right) \cong(\mathbf{Z} / 11 \mathbf{Z})^{2}, \\
& C\left(\mathbf{K}_{60,4}\right) \cong C\left(\mathbf{K}_{5} \mathbf{K}_{13,3}\right) \cong C\left(\mathbf{K}_{13}(\sqrt{5})\right) \cong C\left(\mathbf{K}_{68,8}\right) \cong C\left(\mathbf{K}_{68,4}\right) \\
& \cong C\left(\mathbf{K}_{7}^{+}(\sqrt{-21})\right) \cong C(\mathbf{Q}(\sqrt{-21})) \cong C\left(\mathbf{K}_{7}(\sqrt{13})\right) \cong C\left(\mathbf{K}_{7}^{+} \mathbf{K}_{13,4}\right) \\
& \cong(\mathbf{Z} / 2 \mathbf{Z})^{2} \text {, } \\
& C\left(\mathbf{K}_{65}\right) \cong(\mathbf{Z} / 4 \mathbf{Z})^{2} \oplus(\mathbf{Z} / 2 \mathbf{Z})^{2}, \quad C\left(\mathbf{K}_{5} \mathbf{K}_{13}^{+}\right) \cong C\left(\mathbf{K}_{7}^{+} \mathbf{K}_{11}\right) \cong(\mathbf{Z} / 2 \mathbf{Z})^{4} \text {, } \\
& C\left(\mathbf{K}_{77}\right) \cong(\mathbf{Z} / 4 \mathbf{Z})^{4} \oplus(\mathbf{Z} / 5 \mathbf{Z}), \quad C\left(\mathbf{K}_{7} \mathbf{K}_{11}^{+}\right) \cong(\mathbf{Z} / 2 \mathbf{Z})^{4} \oplus(\mathbf{Z} / 5 \mathbf{Z}), \\
& C\left(\mathbf{K}_{87}\right) \cong(\mathbf{Z} / 8 \mathbf{Z})^{3} \oplus(\mathbf{Z} / 3 \mathbf{Z}), \quad C\left(\mathbf{K}_{29}^{+}(\sqrt{-3})\right) \cong(\mathbf{Z} / 4 \mathbf{Z})^{3} \oplus(\mathbf{Z} / 3 \mathbf{Z}), \\
& C\left(\mathbf{K}_{29,7}(\sqrt{-87})\right) \cong(\mathbf{Z} / 2 \mathbf{Z})^{4} \oplus(\mathbf{Z} / 3 \mathbf{Z}), \\
& C\left(\mathbf{K}_{\mathbf{9 1}}\right) \cong(\mathbf{Z} / \mathbf{4 Z})^{2} \oplus(\mathbf{Z} / \mathbf{7 Z}) \oplus(\mathbf{Z} / \mathbf{1 3 Z}) \oplus(\mathbf{Z} / 37 \mathbf{Z}), \\
& C\left(\mathbf{K}_{7} \mathbf{K}_{13}^{+}\right) \cong(\mathbf{Z} / 2 \mathbf{Z})^{2} \oplus(\mathbf{Z} / 7 \mathbf{Z}) \oplus(\mathbf{Z} / 13 \mathbf{Z}), \quad C\left(\mathbf{K}_{7} \mathbf{K}_{13,4}\right) \cong(\mathbf{Z} / 4 \mathbf{Z})^{2} \text {, } \\
& C\left(\mathbf{K}_{7}^{+} \mathbf{K}_{13}\right) \cong(\mathbf{Z} / 2 \mathbf{Z})^{2} \oplus(\mathbf{Z} / 37 \mathbf{Z}), \\
& C\left(\mathbf{K}_{7}^{+} \mathbf{K}_{13,3}(\sqrt{-91})\right) \cong(\mathbf{Z} / 2 \mathbf{Z})^{3} \oplus(\mathbf{Z} / 7 \mathbf{Z}) \text {, } \\
& C\left(\mathbf{K}_{96}\right) \cong C\left(\mathbf{K}_{32}^{+}(\sqrt{-3})\right) \cong(\mathbf{Z} / 3 \mathbf{Z})^{2}, \\
& C\left(\mathbf{K}_{\mathbf{9 6 , 8}}(\sqrt{-1})\right) \cong C\left(\mathbf{K}_{\mathbf{9 6 , 8}}(\sqrt{3})\right) \cong C\left(\mathbf{K}_{\mathbf{9 6}, 8}\right) \cong(\mathbf{Z} / 2 \mathbf{Z}) \oplus(\mathbf{Z} / 3 \mathbf{Z})^{2} \text {, } \\
& C\left(\mathbf{K}_{\mathbf{9 9}}\right) \cong(\mathbf{Z} / \mathbf{3 Z}) \oplus(\mathbf{Z} / 31 \mathbf{Z})^{2} \text {. }
\end{aligned}
$$

In the last section of the paper, for any imaginary abelian field $k$ in the difference set $\mathscr{H} \backslash\left\{\mathbf{K}_{71}, \mathbf{K}_{73}, \mathbf{K}_{73,24}, \mathbf{K}_{79}, \mathbf{K}_{79,26}, \mathbf{K}_{83}, \mathbf{K}_{89}, \mathbf{K}_{97}, \mathbf{K}_{97,32}\right\}$, we shall see that $C(k)$ is a cyclic group if and only if $C^{-}(l)$ is a cyclic group.

We shall prove I in this section. For simplicity, let $G$ be the Galois group of $K^{+} L$ over $\mathbf{Q}: G=\operatorname{Gal}\left(K^{+} L / \mathbf{Q}\right)$. As usual, let $\mathbf{Z}[G]$ and $\mathbf{Q}[G]$ denote respectively the group ring of $G$ over $\mathbf{Z}$ and the group algebra of $G$ over $\mathbf{Q}$. We regard $\mathbf{Z}[G]$ as a lattice of $\mathrm{Q}[G]$. Let $j$ be the element of $G$ such that, for each $\alpha \in K^{+} L, j(\alpha)$ is the complex conjugate of $\alpha$. Let $\mathfrak{R}$ denote the ideal of $\mathbf{Z}[G]$ generated by $1+j: \Re=(1+j) \mathbf{Z}[G]$. For each subset $Y$ of $G$, denote by $s(Y)$ the sum in $Z[G]$ of all elements of $Y: s(Y)=\sum_{y \in Y} y$. Given 
any prime number $l$, let $T_{l}$ denote the inertia group of $l$ for the extension $K^{+} L / \mathbf{Q}$ and let

$$
\bar{s}_{l}=\frac{1}{\left|T_{l}\right|} \sum_{\lambda} \lambda^{-1},
$$

where $\lambda$ ranges over the Frobenius automorphisms of $l$ for $K^{+} L / \mathbf{Q}$. Note that

$$
T_{p}=\operatorname{Gal}\left(K^{+} L / L\right), \quad T_{q}=\mathrm{Gal}\left(K^{+} L / K^{+}\right) .
$$

We define $\mathfrak{U}$ to be the $\mathfrak{R}$-module generated in $\mathbf{Q}[G]$ by $s(G), s\left(T_{p}\right)\left(1-\bar{s}_{q}\right)$, $s\left(T_{q}\right)\left(1-\bar{s}_{p}\right)$, and $\left(1-\bar{s}_{p}\right)\left(1-\bar{s}_{q}\right)$. It follows that $\mathfrak{U}$ as well as $\mathfrak{R}$ is a lattice of the Q-algebra $(1+j) \mathbf{Q}[G]$ (cf. $\S \S 1,2$ of [14]), so that $\mathfrak{R} \cap \mathfrak{U}$ is also a lattice of $(1+j) \mathbf{Q}[G]$. On the other hand, $j$ does not belong to $T_{p}$ but belongs to $T_{q}$, and from the assumption we see that $G$ coincides with the decomposition group of $q$ for $K^{+} L / \mathbf{Q}$ and that the degree of $q$ for $K^{+} L / \mathbf{Q}$ equals $\left[K^{+}: \mathbf{Q}\right]$. Therefore, by Theorem 5.1 of [14], the generalized index $(\mathfrak{R}: \mathfrak{U})$, i.e., the ratio of the group index $[\mathfrak{R}: \mathfrak{R} \cap \mathfrak{U}]$ to the group index $[\mathfrak{U}: \mathfrak{R} \cap \mathfrak{U}]$ is equal to $2^{1+\delta}$ :

$$
(\mathfrak{R}: \mathfrak{U})=\frac{[\mathfrak{R}: \mathfrak{R} \cap \mathfrak{U}]}{[\mathfrak{U}: \mathfrak{R} \cap \mathfrak{U}]}=2^{1+\delta} .
$$

Now, for each abelian field $k$, let $E(k)$ denote the unit group of $k$ and $E^{*}(k)$ the group of totally positive elements of $k$ in $E(k)$. Since the unit index of $K^{+} L$ equals 1 by "Satz 22" of [4], Theorem 4.1 of [14] implies that

$$
\left[E\left(K^{+} L\right): \operatorname{Cir}\left(K^{+} L\right)\right]=h\left(K^{+} L^{+}\right) 2^{-g}(\mathfrak{R}: \mathfrak{U}), \quad \text { where } g=1 \text { or } 2 .
$$

Furthermore, since $\operatorname{Cir}^{*}\left(K^{+} L\right)=\operatorname{Cir}\left(K^{+} L\right) \cap E^{*}\left(K^{+} L^{+}\right), \operatorname{Cir}^{*}\left(K^{+} L\right)$ is a subgroup of $E^{*}\left(K^{+} L^{+}\right)$with index dividing $\left[E\left(K^{+} L\right): \operatorname{Cir}\left(K^{+} L\right)\right]$;

$$
\left[E^{*}\left(K^{+} L^{+}\right): \operatorname{Cir}^{*}\left(K^{+} L\right)\right] \mid\left[E\left(K^{+} L\right): \operatorname{Cir}\left(K^{+} L\right)\right] .
$$

Consequently,

$$
\left[E^{*}\left(K^{+} L^{+}\right): \operatorname{Cir}^{*}\left(K^{+} L\right)\right] \mid h\left(K^{+} L^{+}\right) 2^{\delta} .
$$

We next put

$$
E_{0}=E\left(K^{+} L^{+}\right) \cap N_{K L^{+} / K^{+} L^{+}}\left(K L^{+}\right) .
$$

Clearly, $E_{0}$ is a subgroup of $E^{*}\left(K^{+} L^{+}\right)$with index dividing $2^{\left[K^{+} L^{+}: \mathbf{Q}\right]}$. It follows from $2 \nmid h\left(K^{+} L^{+}\right)$that $\left\{c \in C\left(K L^{+}\right) \mid c^{2}=1\right\}$ is the set of ambiguous ideal classes in the Sylow 2-subgroup of $C\left(K L^{+}\right)$for the quadratic extension $K L^{+} / K^{+} L^{+}$. Hence the well-known formula for the number of ambiguous ideal classes shows us that

$$
2^{r_{2}\left(K L^{+}\right)}=\frac{2^{t-1+\left[K^{+} L^{+}: \mathbf{Q}\right]}}{\left[E\left(K^{+} L^{+}\right): E_{0}\right]} ;
$$

indeed, $t$ equals the number of prime ideals of $K^{+} L^{+}$ramified in $K L^{+}$. As just one prime ideal of $K^{+} L^{+}$is ramified in $K^{+} L$ by the assumption on $q$, we similarly obtain

$$
2^{r_{2}\left(K^{+} L\right)}=\frac{2^{\left[K^{+} L^{+}: \mathbf{Q}\right]}}{\left[E\left(K^{+} L^{+}\right): E\left(K^{+} L^{+}\right) \cap N_{K^{+} L / K^{+} L^{+}}\left(K^{+} L\right)\right]}
$$

and Hasse's norm theorem and product formula show that

$$
E\left(K^{+} L^{+}\right) \cap N_{K^{+} L / K^{+} L^{+}}\left(K^{+} L\right)=E^{*}\left(K^{+} L^{+}\right) .
$$


Thus

$$
2^{r_{2}\left(K L^{+}\right)}=\frac{2^{r_{2}\left(K^{+} L\right)+t-1}}{\left[E^{*}\left(K^{+} L^{+}\right): E_{0}\right]} .
$$

Now, if $\operatorname{Cir}^{*}\left(K^{+} L\right) \subseteq N_{K L^{+} / K^{+} L^{+}}\left(K L^{+}\right)$, i.e., $\operatorname{Cir}^{*}\left(K^{+} L\right) \subseteq E_{0}$, then (1) implies by $2 \nmid h\left(K^{+} L^{+}\right)$that the 2-power $\left[E^{*}\left(K^{+} L^{+}\right): E_{0}\right]$ divides $2^{\delta}$, so that, by (2), we have

$$
r_{2}\left(K L^{+}\right) \geq r_{2}\left(K^{+} L\right)+t-\delta-1 .
$$

If $\operatorname{Cir}^{*}\left(K^{+} L\right)$ is not contained in $N_{K L^{+} / K^{+} L^{+}}\left(K L^{+}\right)$, namely, there exists an element of $\mathrm{Cir}^{*}\left(K^{+} L\right)$ not contained in $E_{0}$, then obviously $\left[E^{*}\left(K^{+} L^{+}\right): E_{0}\right] \geq$ 2 , and hence it follows again from (2) that

$$
r_{2}\left(K L^{+}\right) \leq r_{2}\left(K^{+} L\right)+t-2 .
$$

Therefore the assertion $\mathrm{I}$ is proved.

The main purpose of the present section is to prove that

$$
C\left(\mathbf{K}_{5} \mathbf{K}_{13}^{+}\right)=C^{-}\left(\mathbf{K}_{5} \mathbf{K}_{13}^{+}\right) \cong(\mathbf{Z} / 2 \mathbf{Z})^{4} \text {. }
$$

From Example 4.1 of [12], we know that $h\left(\mathbf{K}_{65}^{+}\right)=1$. This implies by class field theory that $h\left(\mathbf{K}_{13}^{+}(\sqrt{5})\right)=h(\mathbf{Q}(\sqrt{5}, \sqrt{13}))=1$, since the prime ideal of $\mathbf{Q}(\sqrt{5})$ dividing 13 is totally ramified in $\mathbf{K}_{65}^{+}$. Hence, by "Tafel II" of [4],

$$
h\left(\mathbf{K}_{5} \mathbf{K}_{13}^{+}\right)=16, \quad h\left(\mathbf{K}_{13}(\sqrt{5})\right)=4, \quad h\left(\mathbf{K}_{5}(\sqrt{13})\right)=h\left(\mathbf{K}_{13,4}(\sqrt{5})\right)=1 .
$$

As $\left[K_{5} K_{13}^{+}: K_{5}(\sqrt{13})\right]=\left[K_{13}(\sqrt{5}): K_{13,4}(\sqrt{5})\right]=3$, we then see from Theorem 2.14 of [12] that 2 , the order of 2 modulo 3 , divides both $r_{2}\left(K_{5} K_{13}^{+}\right)$and $r_{2}\left(\mathbf{K}_{13}(\sqrt{5})\right)$, so that

$$
r_{2}\left(\mathbf{K}_{5} \mathbf{K}_{13}^{+}\right)=2 \text { or } 4, \quad r_{2}\left(\mathbf{K}_{13}(\sqrt{5})\right)=2 .
$$

In particular,

$$
C^{-}\left(\mathbf{K}_{13}(\sqrt{5})\right)=C\left(\mathbf{K}_{13}(\sqrt{5})\right) \cong(\mathbf{Z} / 2 \mathbf{Z})^{2} .
$$

Let $D$ be the subgroup of the multiplicative group of $\mathbf{K}_{13}(\sqrt{5})$ generated by the set

$$
\{-1\} \cup\left\{N_{\mathbf{K}_{n} / \mathbf{k}_{n}}\left(1-\zeta_{n}^{a}\right) \mid a \in \mathbf{N}, n \in \mathbf{N}, n \nmid a\right\},
$$

where $\mathbf{k}_{n}=\mathbf{K}_{13}(\sqrt{5}) \cap \mathbf{K}_{n}$ for each $n \in \mathbf{N}$. Then we easily see that the subset of the above,

$$
\{-1\} \cup\left\{N_{\mathbf{K}_{n} / \mathbf{k}_{n}}\left(1-\zeta_{n}^{a}\right) \mid a \in \mathbf{N} ; n=5,13 \text { or } 65 ;(a, n)=1\right\},
$$

already generates $D$. Since

$$
\operatorname{Cir}\left(\mathbf{K}_{13}(\sqrt{5})\right)=D \cap E\left(\mathbf{K}_{13}(\sqrt{5})\right)
$$

by definition (cf. $\S 4$ of [14]), it follows that $\operatorname{Cir}\left(\mathbf{K}_{13}(\sqrt{5})\right.$ ) is generated by

$$
\begin{aligned}
& \left\{-\zeta_{13},\left(1+\zeta_{5}\right)\left(1+\zeta_{5}^{-1}\right)\right\} \\
& \quad \cup\left\{\left(1-\zeta_{65}^{a}\right)\left(1-\zeta_{65}^{14 a}\right) \mid a \in \mathbf{N}, a \leq 32,(a, 65)=1\right\} \\
& \quad \cup\left\{\frac{1-\zeta_{13}^{a}}{1-\zeta_{13}} \mid a \in \mathbf{N}, 2 \leq a \leq 6\right\} .
\end{aligned}
$$


Furthermore, since $\left(1-\zeta_{65}^{a}\right)\left(1-\zeta_{65}^{14 a}\right) \zeta_{13}^{5 a}$ and $\left(1-\zeta_{13}^{a}\right)\left(1-\zeta_{13}\right)^{-1} \zeta_{13}^{6 a-6}$ are real for each $a \in \mathbf{N}, \operatorname{Cir}\left(\mathbf{K}_{13}(\sqrt{5})\right)$ is the direct product of $\operatorname{Cir}^{+}\left(\mathbf{K}_{13}(\sqrt{5})\right)$ and the cyclic group $\left\langle\zeta_{13}\right\rangle$. Thus we know that a set of generators for $\operatorname{Cir}^{+}\left(\mathbf{K}_{13}(\sqrt{5})\right)$ consists of $-1,\left(1+\zeta_{5}\right)\left(1+\zeta_{5}^{-1}\right)=((1+\sqrt{5}) / 2)^{2}$, and the 17 units $\varepsilon_{1}, \ldots, \varepsilon_{17}$ of $\mathbf{K}_{13}^{+}(\sqrt{5})$ defined by

$$
\begin{array}{ll}
\varepsilon_{1}=\left(1-\zeta_{65}\right)\left(1-\zeta_{65}^{14}\right) \zeta_{13}^{5}, & \varepsilon_{2}=\left(1-\zeta_{65}^{2}\right)\left(1-\zeta_{65}^{28}\right) \zeta_{13}^{-3}, \\
\varepsilon_{3}=\left(1-\zeta_{65}^{3}\right)\left(1-\zeta_{65}^{-23}\right) \zeta_{13}^{2}, & \varepsilon_{4}=\left(1-\zeta_{65}^{4}\right)\left(1-\zeta_{65}^{-9}\right) \zeta_{13}^{-6}, \\
\varepsilon_{5}=\left(1-\zeta_{65}^{6}\right)\left(1-\zeta_{65}^{19}\right) \zeta_{13}^{4}, & \varepsilon_{6}=\left(1-\zeta_{65}^{7}\right)\left(1-\zeta_{65}^{-32}\right) \zeta_{13}^{-4}, \\
\varepsilon_{7}=\left(1-\zeta_{65}^{8}\right)\left(1-\zeta_{65}^{-18}\right) \zeta_{13}, & \varepsilon_{8}=\left(1-\zeta_{65}^{11}\right)\left(1-\zeta_{65}^{24}\right) \zeta_{13}^{3}, \\
\varepsilon_{9}=\left(1-\zeta_{65}^{12}\right)\left(1-\zeta_{65}^{-27}\right) \zeta_{13}^{-5}, & \varepsilon_{10}=\left(1-\zeta_{65}^{16}\right)\left(1-\zeta_{65}^{29}\right) \zeta_{13}^{2}, \\
\varepsilon_{11}=\left(1-\zeta_{65}^{17}\right)\left(1-\zeta_{65}^{-22}\right) \zeta_{13}^{-6}, \quad \varepsilon_{12}=\left(1-\zeta_{65}^{21}\right)\left(1-\zeta_{65}^{-31}\right) \zeta_{13}, \\
\varepsilon_{13}=\frac{1-\zeta_{13}^{2}}{1-\zeta_{13}} \zeta_{13}^{6}, \quad \varepsilon_{14}=\frac{1-\zeta_{13}^{3}}{1-\zeta_{13}} \zeta_{13}^{-1}, \quad \varepsilon_{15}=\frac{1-\zeta_{13}^{4}}{1-\zeta_{13}} \zeta_{13}^{5}, \\
\varepsilon_{16}=\frac{1-\zeta_{13}^{5}}{1-\zeta_{13}} \zeta_{13}^{-2}, \quad \varepsilon_{17}=\frac{1-\zeta_{13}^{6}}{1-\zeta_{13}} \zeta_{13}^{4} .
\end{array}
$$

Let $f_{1}, \ldots, f_{12}$ be the elements of $\operatorname{Gal}\left(\mathbf{K}_{65} / \mathbf{Q}\right)$ such that

$$
\begin{array}{llll}
f_{1}\left(\zeta_{65}\right)=\zeta_{65}, & f_{2}\left(\zeta_{65}\right)=\zeta_{65}^{2}, & f_{3}\left(\zeta_{65}\right)=\zeta_{65}^{6}, & f_{4}\left(\zeta_{65}\right)=\zeta_{65}^{9}, \\
f_{5}\left(\zeta_{65}\right)=\zeta_{65}^{16}, & f_{6}\left(\zeta_{65}\right)=\zeta_{65}^{17}, & f_{7}\left(\zeta_{65}\right)=\zeta_{65}^{18}, & f_{8}\left(\zeta_{65}\right)=\zeta_{65}^{21}, \\
f_{9}\left(\zeta_{65}\right)=\zeta_{65}^{23}, & f_{10}\left(\zeta_{65}\right)=\zeta_{65}^{24}, & f_{11}\left(\zeta_{65}\right)=\zeta_{65}^{27}, & f_{12}\left(\zeta_{65}\right)=\zeta_{65}^{32} .
\end{array}
$$

It then follows that $\operatorname{Gal}\left(\mathbf{K}_{13}^{+}(\sqrt{5}) / \mathbf{Q}\right)$ consists of the restrictions of all $f_{a}, 1 \leq$ $a \leq 12$, on $\mathbf{K}_{13}^{+}(\sqrt{5})$. Let $\mathbf{F}_{2}$ denote as usual the field whose additive group is Z/2Z. Putting

$$
\overline{0}=2 \mathbf{Z}, \quad \overline{1}=\{1+2 n \mid n \in \mathbf{Z}\},
$$

let $M$ be the matrix over $F_{2}$ of $(12,18)$-type such that every component of the 18-th column of $M$ equals 1 and, for each $a, b \in \mathbf{N}$ with $a \leq 12$ and $b \leq 17$, the $(a, b)$-component of $M$ equals $\overline{0}$ or $\overline{1}$ according as $f_{a}\left(\varepsilon_{b}\right)$ is positive or negative. For each $a, b \in \mathbf{N}$ with $a \leq 18, b \leq 18$ and $a \neq b$, let $R_{a, b}$ denote the square matrix over $\mathbf{F}_{2}$ of degree 18 such that the diagonal components and the $(a, b)$-component of $R_{a, b}$ equal $\overline{1}$ and the other components of $R_{a, b}$ equal $\overline{0}$. Let

$$
\begin{aligned}
& R_{11}=R_{11,2} R_{11,4} R_{11,6} R_{11,9} R_{11,13} R_{11,15} R_{11,17} R_{11,18}, \\
& R_{5}=R_{5,1} R_{5,2} R_{5,8} R_{5,10} R_{5,18} \\
& R_{12}=R_{12,4} R_{12,6} R_{12,7} R_{12,13} R_{12,14} R_{12,17} \\
& R_{3}=R_{3,1} R_{3,6} R_{3,7} R_{3,9} R_{3,15} R_{3,16} R_{3,17} R_{3,18} \\
& R_{10}=R_{10,1} R_{10,4} R_{10,15} R_{10,18} \\
& R_{9}=R_{9,2} R_{9,4} R_{9,6} R_{9,7} R_{9,8} R_{9,13} R_{9,14} R_{9,15} R_{9,16} \\
& R_{8}=R_{8,1} R_{8,2} R_{8,7} R_{8,16}, \\
& R_{7}=R_{7,2} R_{7,6} R_{7,14} R_{7,15} R_{7,16} R_{7,17} R_{7,18} \\
& R_{2}=R_{2,13} R_{2,18} \\
& R_{0}=R_{11} R_{5} R_{12} R_{3} R_{10} R_{9} R_{8} R_{7} R_{14,13} R_{17,15} R_{2} R_{4,15}
\end{aligned}
$$


Then

$$
M R_{0}=\left(\begin{array}{cccccccccccccccccc}
\overline{0} & \overline{0} & \overline{0} & \overline{0} & \overline{0} & \overline{0} & \overline{0} & \overline{0} & \overline{0} & \overline{0} & \overline{1} & \overline{0} & \overline{0} & \overline{0} & \overline{0} & \overline{0} & \overline{0} & \overline{0} \\
\overline{0} & \overline{0} & \overline{0} & \overline{0} & \overline{1} & \overline{0} & \overline{0} & \overline{0} & \overline{0} & \overline{0} & \overline{0} & \overline{0} & \overline{0} & \overline{0} & \overline{0} & \overline{0} & \overline{0} & \overline{0} \\
\overline{0} & \overline{0} & \overline{0} & \overline{0} & \overline{0} & \overline{0} & \overline{0} & \overline{0} & \overline{0} & \overline{0} & \overline{1} & \overline{1} & \overline{0} & \overline{0} & \overline{0} & \overline{0} & \overline{0} & \overline{0} \\
\overline{0} & \overline{0} & \overline{1} & \overline{0} & \overline{0} & \overline{0} & \overline{0} & \overline{0} & \overline{0} & \overline{0} & \overline{0} & \overline{0} & \overline{0} & \overline{0} & \overline{0} & \overline{0} & \overline{0} & \overline{0} \\
\overline{0} & \overline{0} & \overline{1} & \overline{0} & \overline{0} & \overline{0} & \overline{0} & \overline{0} & \overline{0} & \overline{1} & \overline{1} & \overline{0} & \overline{0} & \overline{0} & \overline{0} & \overline{0} & \overline{0} & \overline{0} \\
\overline{0} & \overline{0} & \overline{0} & \overline{0} & \overline{1} & \overline{0} & \overline{0} & \overline{0} & \overline{1} & \overline{0} & \overline{0} & \overline{1} & \overline{0} & \overline{0} & \overline{0} & \overline{0} & \overline{0} & \overline{0} \\
\overline{0} & \overline{0} & \overline{0} & \overline{0} & \overline{1} & \overline{0} & \overline{0} & \overline{1} & \overline{0} & \overline{0} & \overline{0} & \overline{1} & \overline{0} & \overline{0} & \overline{0} & \overline{0} & \overline{0} & \overline{0} \\
\overline{0} & \overline{0} & \overline{1} & \overline{0} & \overline{1} & \overline{0} & \overline{1} & \overline{1} & \overline{0} & \overline{1} & \overline{1} & \overline{0} & \overline{0} & \overline{0} & \overline{0} & \overline{0} & \overline{0} & \overline{0} \\
\overline{0} & \overline{0} & \overline{1} & \overline{0} & \overline{0} & \overline{0} & \overline{1} & \overline{0} & \overline{1} & \overline{1} & \overline{0} & \overline{1} & \overline{0} & \overline{1} & \overline{0} & \overline{0} & \overline{1} & \overline{0} \\
\overline{0} & \overline{0} & \overline{1} & \overline{0} & \overline{0} & \overline{0} & \overline{0} & \overline{1} & \overline{0} & \overline{0} & \overline{0} & \overline{0} & \overline{0} & \overline{0} & \overline{0} & \overline{0} & \overline{1} & \overline{0} \\
\overline{0} & \overline{0} & \overline{0} & \overline{0} & \overline{1} & \overline{0} & \overline{0} & \overline{1} & \overline{1} & \overline{1} & \overline{1} & \overline{0} & \overline{0} & \overline{1} & \overline{0} & \overline{0} & \overline{0} & \overline{0} \\
\overline{0} & \overline{0} & \overline{0} & \overline{0} & \overline{0} & \overline{0} & \overline{1} & \overline{1} & \overline{0} & \overline{0} & \overline{0} & \overline{1} & \overline{0} & \overline{1} & \overline{0} & \overline{0} & \overline{0} & \overline{0}
\end{array}\right),
$$

and, for any $x_{1}, \ldots, x_{18} \in F_{2}$, the first, second, 4th, 6th, 13th, 15th, 16th, and 18th components of the row vector $\left(x_{1}, \ldots, x_{18}\right) R_{0}$ are $x_{1}+x_{5}+x_{8}+$ $x_{9}+x_{10}+x_{11}, x_{2}+x_{3}+x_{5}+x_{7}+x_{11}+x_{12}, x_{3}+x_{4}+x_{5}+x_{9}+x_{10}+x_{12}$, $x_{3}+x_{5}+x_{6}+x_{7}+x_{8}+x_{9}, x_{2}+x_{8}+x_{13}+x_{14}, x_{4}+x_{11}+x_{15}+x_{17}, x_{7}+x_{12}+x_{16}$, and $x_{2}+x_{3}+x_{8}+x_{10}+x_{18}$, respectively. We therefore see that $\operatorname{Cir}^{*}\left(\mathbf{K}_{13}(\sqrt{5})\right)$ is generated by

$$
\begin{aligned}
& \left\{\eta^{2} \mid \eta \in \operatorname{Cir}^{+}\left(\mathbf{K}_{13}(\sqrt{5})\right)\right\} \\
& \cup\left\{\varepsilon_{1} \varepsilon_{5} \varepsilon_{8} \varepsilon_{9} \varepsilon_{10} \varepsilon_{11}, \varepsilon_{2} \varepsilon_{3} \varepsilon_{5} \varepsilon_{7} \varepsilon_{11} \varepsilon_{12}, \quad \varepsilon_{3} \varepsilon_{4} \varepsilon_{5} \varepsilon_{9} \varepsilon_{10} \varepsilon_{12},\right. \\
& \\
& \left.\quad \varepsilon_{3} \varepsilon_{5} \varepsilon_{6} \varepsilon_{7} \varepsilon_{8} \varepsilon_{9}, \varepsilon_{2} \varepsilon_{8} \varepsilon_{13} \varepsilon_{14}, \varepsilon_{4} \varepsilon_{11} \varepsilon_{15} \varepsilon_{17}, \varepsilon_{7} \varepsilon_{12} \varepsilon_{16},-\varepsilon_{2} \varepsilon_{3} \varepsilon_{8} \varepsilon_{10}\right\} .
\end{aligned}
$$

Let $\alpha=\zeta_{13}+\zeta_{13}^{-1}$ and $\beta=\zeta_{5}+\zeta_{5}^{-1}$. Then

$$
\alpha^{6}=1-3 \alpha-6 \alpha^{2}+4 \alpha^{3}+5 \alpha^{4}-\alpha^{5}, \quad \beta \equiv 2(\bmod \sqrt{5}),
$$

and $\left\{1, \alpha, \alpha^{2}, \alpha^{3}, \alpha^{4}, \alpha^{5}, \beta, \alpha \beta, \alpha^{2} \beta, \alpha^{3} \beta, \alpha^{4} \beta, \alpha^{5} \beta\right\}$ is a basis of the additive group of algebraic integers in $\mathbf{K}_{13}^{+}(\sqrt{5})$. On the other hand,

$$
\begin{gathered}
f_{7}\left(\varepsilon_{1} \varepsilon_{5} \varepsilon_{8} \varepsilon_{9} \varepsilon_{10} \varepsilon_{11}\right)=f_{12}\left(\varepsilon_{3} \varepsilon_{4} \varepsilon_{5} \varepsilon_{9} \varepsilon_{10} \varepsilon_{12}\right)=f_{5}\left(\varepsilon_{3} \varepsilon_{5} \varepsilon_{6} \varepsilon_{7} \varepsilon_{8} \varepsilon_{9}\right)=\varepsilon_{2} \varepsilon_{3} \varepsilon_{5} \varepsilon_{7} \varepsilon_{11} \varepsilon_{12}, \\
f_{7}^{2}=f_{1}=1, \quad f_{12} f_{2}=1, \quad f_{5} f_{4}=1 .
\end{gathered}
$$

Using these facts, we have

$$
\begin{array}{rlrl}
\varepsilon_{2} \varepsilon_{3} \varepsilon_{5} \varepsilon_{7} \varepsilon_{11} \varepsilon_{12} & \equiv\left(\alpha^{3}+\alpha^{4}+\alpha^{5}\right)^{2} & & (\bmod \sqrt{5}) \\
\varepsilon_{1} \varepsilon_{5} \varepsilon_{8} \varepsilon_{9} \varepsilon_{10} \varepsilon_{11} & \equiv\left(f_{7}\left(\alpha^{3}+\alpha^{4}+\alpha^{5}\right)\right)^{2} & & (\bmod \sqrt{5}), \\
\varepsilon_{3} \varepsilon_{4} \varepsilon_{5} \varepsilon_{9} \varepsilon_{10} \varepsilon_{12} & \equiv\left(f_{2}\left(\alpha^{3}+\alpha^{4}+\alpha^{5}\right)\right)^{2} & & (\bmod \sqrt{5}) \\
\varepsilon_{3} \varepsilon_{5} \varepsilon_{6} \varepsilon_{7} \varepsilon_{8} \varepsilon_{9} & \equiv\left(f_{4}\left(\alpha^{3}+\alpha^{4}+\alpha^{5}\right)\right)^{2} & & (\bmod \sqrt{5}) \\
\varepsilon_{2} \varepsilon_{8} \varepsilon_{13} \varepsilon_{14} & \equiv\left(1+2 \alpha^{3}+4 \alpha^{4}+3 \alpha^{5}\right)^{2} & (\bmod \sqrt{5}) \\
\varepsilon_{4} \varepsilon_{11} \varepsilon_{15} \varepsilon_{17} & \equiv\left(1+2 \alpha+\alpha^{2}+3 \alpha^{5}\right)^{2} & & (\bmod \sqrt{5}) \\
\varepsilon_{7} \varepsilon_{12} \varepsilon_{16} & \equiv\left(4+\alpha+\alpha^{2}\right)^{2} & & (\bmod \sqrt{5}) \\
-\varepsilon_{2} \varepsilon_{3} \varepsilon_{8} \varepsilon_{10} & \equiv 1 & & (\bmod \sqrt{5}) .
\end{array}
$$

Since the conductor of $\mathbf{K}_{5} \mathbf{K}_{13}^{+}$over $\mathbf{K}_{13}^{+}(\sqrt{5})$ is the product of all infinite primes of $\mathbf{K}_{13}^{+}(\sqrt{5})$ and the principal ideal $(\sqrt{5})$, the Hasse norm theorem implies for 
any $\eta \in E^{*}\left(\mathbf{K}_{13}^{+}(\sqrt{5})\right)$ that if $\eta \equiv \xi^{2}(\bmod \sqrt{5})$ with some algebraic integer $\xi$ in $\mathbf{K}_{13}^{+}(\sqrt{5})$, then $\eta$ lies in $N_{\mathbf{K}_{5} \mathbf{K}_{13}^{+} / \mathbf{K}_{13}^{+}(\sqrt{5})}\left(\mathbf{K}_{5} \mathbf{K}_{13}^{+}\right)$. Thus we have

$$
\operatorname{Cir}^{*}\left(\mathbf{K}_{13}(\sqrt{5})\right) \subseteq N_{\mathbf{K}_{5} \mathbf{K}_{13}^{+} / \mathbf{K}_{13}^{+}(\sqrt{5})}\left(\mathbf{K}_{5} \mathbf{K}_{13}^{+}\right) .
$$

Furthermore, $h\left(\mathbf{K}_{13}^{+}(\sqrt{5})\right)=1$ as already mentioned, just three distinct prime ideals of $\mathbf{K}_{13}^{+}(\sqrt{5})$ divide $5, \mathbf{K}_{5}^{+}=\mathbf{Q}(\sqrt{5})$ is a quadratic field, and 13 is not decomposed in $K_{65}=K_{5} K_{13}$. It therefore follows from $I$ that

$$
r_{2}\left(\mathbf{K}_{5} \mathbf{K}_{13}^{+}\right) \geq r_{2}\left(\mathbf{K}_{13}(\sqrt{5})\right)+1,
$$

so that (3) shows that $r_{2}\left(\mathbf{K}_{5} \mathbf{K}_{13}^{+}\right)=4$. This now means, by $h\left(\mathbf{K}_{5} \mathbf{K}_{13}^{+}\right)=16$, that

$$
C^{-}\left(\mathbf{K}_{5} \mathbf{K}_{13}^{+}\right)=C\left(\mathbf{K}_{5} \mathbf{K}_{13}^{+}\right) \cong(\mathbf{Z} / 2 \mathbf{Z})^{4} \text {. }
$$

Note next that, since $h\left(\mathbf{K}_{65}^{+}\right)=h\left(\mathbf{K}_{13}^{+}(\sqrt{5})\right)=h(\mathbf{Q}(\sqrt{5}, \sqrt{13}))=1$, we easily have $h\left(K^{+}\right)=1$ for every imaginary abelian field $K$ with $f(K)=65$. For instance, we have $h\left(\mathbf{K}_{13,3}(\sqrt{5})\right)=1$ by the fact that the prime ideal of $\mathbf{K}_{13,3}(\sqrt{5})$ dividing 13 is ramified in the quadratic extension $\mathbf{K}_{13}^{+}(\sqrt{5})$ over $\mathbf{K}_{13,3}(\sqrt{5})$. We further know from "Tafel II" of [4] that the imaginary abelian fields with conductor 65 , different from $\mathbf{K}_{5} \mathbf{K}_{13}^{+}, \mathbf{K}_{13}(\sqrt{5}), \mathbf{K}_{65}, \mathbf{K}_{5} \mathbf{K}_{13,3}, \mathbf{L}_{65,12}, \mathbf{L}_{65,12}^{\prime}$, have square-free relative class numbers and that

$$
h^{-}\left(\mathbf{K}_{65}\right)=2^{6}, \quad h^{-}\left(\mathbf{K}_{5} \mathbf{K}_{13,3}\right)=2^{2}, \quad h^{-}\left(\mathbf{L}_{65,12}\right)=2^{3}, \quad h^{-}\left(\mathbf{L}_{65,12}^{\prime}\right)=2^{3} .
$$

It is shown in [5] that

$$
C\left(\mathbf{K}_{65}\right)=C^{-}\left(\mathbf{K}_{65}\right) \cong(\mathbf{Z} / 4 \mathbf{Z})^{2} \oplus(\mathbf{Z} / 2 \mathbf{Z})^{2} .
$$

By $h\left(\mathbf{K}_{5}\right)=1$ and $\left[\mathbf{K}_{5} \mathbf{K}_{13,3}: \mathbf{K}_{5}\right]=3$, Theorem 2.14 of [12] implies

$$
C^{-}\left(\mathbf{K}_{5} \mathbf{K}_{13,3}\right)=C\left(\mathbf{K}_{5} \mathbf{K}_{13,3}\right) \cong(\mathbf{Z} / 2 \mathbf{Z})^{2} \text {. }
$$

This fact follows also from $C\left(\mathbf{K}_{5} \mathbf{K}_{13}^{+}\right) \cong(\mathbf{Z} / 2 \mathbf{Z})^{4}$. Now let

$$
\omega=-\left(\zeta_{13}+\zeta_{13}^{-1}\right)\left(\zeta_{13}^{5}+\zeta_{13}^{-5}\right)-1
$$

so that

$$
\omega \in \mathbf{K}_{13,3}, \quad \omega^{3}+2 \omega^{2}-3 \omega-5=0 .
$$

Let $\sigma$ be a generator of the cyclic group $\operatorname{Gal}\left(\mathbf{K}_{13,3} / \mathbf{Q}\right)$. As the prime 5 is completely decomposed in $\mathbf{K}_{13,3}$, the principal ideals $(\omega),\left(\omega^{\sigma}\right),\left(\omega^{\sigma^{2}}\right)$ are the three distinct prime ideals dividing 5. By $\omega+1 \in E\left(\mathbf{K}_{13,3}\right)$ and $\omega \equiv(\omega+1)^{-2}(\bmod 4)$, the prime ideals of $\mathbf{K}_{13,3}$ dividing 2 are unramified in $\mathbf{K}_{13,3}\left(\sqrt{\omega}, \sqrt{\omega^{\sigma}}\right)$. On the other hand, $\mathbf{L}_{65,12}$ is a quartic extension over $\mathbf{K}_{13,3}$ in which $(\omega)$ and $\left(\omega^{\sigma}\right)$ are fully ramified. Hence $\mathbf{L}_{65,12}\left(\sqrt{\omega}, \sqrt{\omega^{\sigma}}\right)$ is unramified over $\mathbf{L}_{65,12}$. We also see from the relations

$$
\omega \omega^{\sigma}+\omega^{\sigma} \omega^{\sigma^{2}}+\omega^{\sigma^{2}} \omega=-3, \quad \omega \omega^{\sigma} \omega^{\sigma^{2}}=5
$$

that each of $\sqrt{\omega}, \sqrt{\omega^{\sigma}}, \sqrt{\omega \omega^{\sigma}}$ is neither totally real nor totally imaginary. Furthermore, by genus theory, $\mathbf{K}_{5} \mathbf{K}_{13}^{+}$is an unramified quadratic extension over $\mathbf{L}_{65,12}$. Thus $\mathbf{K}_{5} \mathbf{K}_{13}^{+}\left(\sqrt{\omega}, \sqrt{\omega^{\sigma}}\right)$ is an unramified abelian extension over $\mathbf{L}_{65,12}$, and

$$
\operatorname{Gal}\left(\mathbf{K}_{5} \mathbf{K}_{13}^{+}\left(\sqrt{\omega}, \sqrt{\omega^{\sigma}}\right) / \mathbf{L}_{65,12}\right) \cong(\mathbf{Z} / 2 \mathbf{Z})^{3} \text {. }
$$


Since $h^{-}\left(\mathbf{L}_{65,12}\right)=h\left(\mathbf{L}_{65,12}\right)=2^{3}$, class field theory then shows that

$$
C^{-}\left(\mathbf{L}_{65}, 12\right)=C\left(\mathbf{L}_{65,12}\right) \cong(\mathbf{Z} / 2 \mathbf{Z})^{3} \text {. }
$$

Note that $\mathbf{K}_{5} \mathbf{K}_{13}^{+}\left(\sqrt{\omega}, \sqrt{\omega^{\sigma}}\right)$ is the Hilbert class field over $\mathbf{L}_{65,12}$. Similarly, we can see that $\mathbf{K}_{13}\left(\sqrt{5}, \sqrt{\omega}, \sqrt{\omega^{\sigma}}\right)$ is the Hilbert class field over $\mathbf{L}_{65,12}^{\prime}$, as well as that

$$
C^{-}\left(\mathbf{L}_{65,12}^{\prime}\right)=C\left(\mathbf{L}_{65,12}^{\prime}\right) \cong(\mathbf{Z} / 2 \mathbf{Z})^{3} .
$$

Let us prove the assertion II. The discussion in the preceding section guarantees that it now suffices to consider the imaginary abelian fields $k$ in $\mathscr{H}$ with $f(k) \neq 65$. The values of the relative class numbers of all imaginary abelian fields in $\mathscr{H}$ are given by "Tafel II" of [4]. In particular, we see that an imaginary abelian field in $\mathscr{H}$ whose conductor differs from 65 does not have square-free relative class number if and only if it is one of the following 59 fields:

$\mathbf{K}_{29}, \mathbf{K}_{31}, \mathbf{K}_{31,6}, \mathbf{K}_{41}, \mathbf{K}_{71}, \mathbf{K}_{71,14}, \mathbf{K}_{13,3}(\sqrt{-39}), \mathbf{Q}(\sqrt{-39}), \mathbf{K}_{11}^{+}(\sqrt{-55})$, $\mathbf{Q}(\sqrt{-55}), \mathbf{K}_{7}^{+}(\sqrt{-1}, \sqrt{-14}), \mathbf{Q}(\sqrt{-1}, \sqrt{-14}), \mathbf{K}_{7}^{+}(\sqrt{-2}, \sqrt{-14})$, $\mathbf{Q}(\sqrt{-2}, \sqrt{-14}), \mathbf{K}_{7}^{+}(\sqrt{-14}), \mathbf{Q}(\sqrt{-14}), \mathbf{K}_{57}, \mathbf{K}_{19}^{+}(\sqrt{-3}), \mathbf{K}_{60,4}, \mathbf{K}_{68}$, $\mathbf{K}_{17}^{+}(\sqrt{-1}), \mathbf{K}_{17,4}(\sqrt{-1}), \mathbf{K}_{68,8}, \mathbf{K}_{68,4}, \mathbf{Q}(\sqrt{-17}), \mathbf{K}_{77}, \mathbf{K}_{7} \mathbf{K}_{11}^{+}, \mathbf{K}_{7}^{+} \mathbf{K}_{11}$, $\mathbf{K}_{7}^{+}(\sqrt{-21}), \mathbf{Q}(\sqrt{-21}), \mathbf{K}_{87}, \mathbf{K}_{29}^{+}(\sqrt{-3}), \mathbf{K}_{29,7}(\sqrt{-3}), \mathbf{K}_{29,7}(\sqrt{-87})$, $\mathbf{K}_{91}, \mathbf{K}_{7} \mathbf{K}_{13}^{+}, \mathbf{K}_{7} \mathbf{K}_{13,4}, \mathbf{K}_{7}(\sqrt{13}), \mathbf{K}_{7}^{+} \mathbf{K}_{13}, \mathbf{K}_{7}^{+} \mathbf{K}_{13,4}, \mathbf{K}_{7}^{+} \mathbf{K}_{13,3}(\sqrt{-91})$, $\mathbf{K}_{7}^{+}(\sqrt{-91}), \mathbf{K}_{93}, \mathbf{K}_{31,6}(\sqrt{-3}), \mathbf{K}_{95}, \mathbf{K}_{5} \mathbf{K}_{19,6}, \mathbf{K}_{5}(\sqrt{-19}), \mathbf{K}_{19}(\sqrt{5})$, $\mathbf{K}_{19,6}(\sqrt{5}), \mathbf{Q}(\sqrt{5}, \sqrt{-19}), \mathbf{K}_{19}^{+}(\sqrt{-95}), \mathbf{K}_{19,3}(\sqrt{-95}), \mathbf{Q}(\sqrt{-95}), \mathbf{K}_{96}$, $\mathbf{K}_{32}^{+}(\sqrt{-3}), \mathbf{K}_{96,8}(\sqrt{-1}), \mathbf{K}_{96,8}(\sqrt{3}), \mathbf{K}_{96,8}, \mathbf{K}_{99}$.

We further know from the results of $[1,11,12]$ that, except $\mathbf{K}_{71}^{+}$, the maximal real subfield of each of these fields has class number 1 . We keep such datum and the next trivial lemma in mind throughout the rest of the paper.

Lemma (cf. Lemma 7 of [15]). Let $F / k$ be an extension of imaginary abelian fields and let $p$ be a prime number. Let $S_{F}$ denote the Sylow $p$-subgroup of $C^{-}(F), S_{k}$ the Sylow p-subgroup of $C^{-}(k), I$ the natural map $C(k) \rightarrow C(F)$, and $\nu$ the norm map $C(F) \rightarrow C(k)$. Assume that $p$ does not divide $[F: k]$. Then $I$ induces an injective homomorphism $S_{k} \rightarrow S_{F}, \nu$ induces a surjective homomorphism $S_{F} \rightarrow S_{k}$, and $S_{F}=I\left(S_{k}\right) \times\left(\operatorname{Ker} \nu \cap S_{F}\right)$.

As stated in [9, 10] and justified in [7], etc.,

$$
\begin{gathered}
C^{-}\left(\mathbf{K}_{29}\right) \cong(\mathbf{Z} / 2 \mathbf{Z})^{3}, \quad C^{-}\left(\mathbf{K}_{31}\right) \cong \mathbf{Z} / 9 \mathbf{Z}, \quad C^{-}\left(\mathbf{K}_{41}\right) \cong(\mathbf{Z} / 11 \mathbf{Z})^{2}, \\
C^{-}\left(\mathbf{K}_{71}\right) \cong(\mathbf{Z} / 49 \mathbf{Z}) \oplus(\mathbf{Z} / 79241 \mathbf{Z}) .
\end{gathered}
$$

On the other hand,

$$
\begin{gathered}
h\left(\mathbf{K}_{29}^{+}\right)=h\left(\mathbf{K}_{41}^{+}\right)=1, \quad h^{-}\left(\mathbf{K}_{31,6}\right)=9, \quad h^{-}\left(\mathbf{K}_{71,14}\right)=49, \\
{\left[\mathbf{K}_{31}: \mathbf{K}_{31,6}\right]=\left[\mathbf{K}_{71}: \mathbf{K}_{71,14}\right]=5 .}
\end{gathered}
$$


It therefore follows that

$$
\begin{array}{ll}
C\left(\mathbf{K}_{29}\right) \cong(\mathbf{Z} / 2 \mathbf{Z})^{3}, & C\left(\mathbf{K}_{41}\right) \cong(\mathbf{Z} / 11 \mathbf{Z})^{2}, \quad C^{-}\left(\mathbf{K}_{31,6}\right) \cong \mathbf{Z} / 9 \mathbf{Z}, \\
& C^{-}\left(\mathbf{K}_{71}, 14\right) \cong \mathbf{Z} / 49 \mathbf{Z}
\end{array}
$$

(cf. the lemma; for the result $C\left(\mathbf{K}_{29}\right) \cong(\mathbf{Z} / 2 \mathbf{Z})^{3}$, see also [2, 3]). Now, we easily have $C(\mathbf{Q}(\sqrt{-39})) \cong \mathbf{Z} / 4 \mathbf{Z}$, so that

$$
C^{-}\left(\mathbf{K}_{13,3}(\sqrt{-39})\right) \cong \mathbf{Z} / 4 \mathbf{Z}
$$

by $h^{-}\left(\mathbf{K}_{13,3}(\sqrt{-39})\right)=4$ and $\left[\mathbf{K}_{13,3}(\sqrt{-39}): \mathbf{Q}(\sqrt{-39})\right]=3$. Similarly, we have

$$
\begin{aligned}
& C(\mathbf{Q}(\sqrt{-55})) \cong C^{-}\left(\mathbf{K}_{11}^{+}(\sqrt{-55})\right) \cong \mathbf{Z} / 4 \mathbf{Z}, \\
& C(\mathbf{Q}(\sqrt{-14})) \cong C^{-}\left(\mathbf{K}_{7}^{+}(\sqrt{-14})\right) \cong \mathbf{Z} / 4 \mathbf{Z} .
\end{aligned}
$$

It further follows for either $a \in\{1,2\}$ that

$$
\begin{aligned}
C^{-}\left(\mathbf{K}_{7}^{+}(\sqrt{-a}, \sqrt{-14})\right) & \cong C^{-}(\mathbf{Q}(\sqrt{-a}, \sqrt{-14}))=C(\mathbf{Q}(\sqrt{-a}, \sqrt{-14})) \\
& \cong \mathbf{Z} / 4 \mathbf{Z},
\end{aligned}
$$

since $h^{-}(\mathbf{Q}(\sqrt{-a}, \sqrt{-14}))=h^{-}\left(\mathbf{K}_{7}^{+}(\sqrt{-a}, \sqrt{-14})\right)=4, \mathbf{K}_{7}^{+}(\sqrt{-a}, \sqrt{-14})$ is a cubic extension over $\mathbf{Q}(\sqrt{-a}, \sqrt{-14})$, and the prime ideal of $\mathbf{Q}(\sqrt{-14})$ dividing 2 is ramified in $\mathbf{Q}(\sqrt{-a}, \sqrt{-14})$.

Note next that $h\left(\mathbf{K}_{19,3}\right)=1$ and that $h^{-}\left(\mathbf{K}_{19}^{+}(\sqrt{-3})\right)\left(=h\left(\mathbf{K}_{19}^{+}(\sqrt{-3})\right)\right)=9$. Let $\Gamma=\operatorname{Gal}\left(\mathbf{K}_{19}^{+}(\sqrt{-3}) / \mathbf{K}_{19,3}(\sqrt{-3})\right)$ and let $\gamma$ be a non-trivial element of $\Gamma$. Since $\Gamma$ acts on $C\left(\mathbf{K}_{19}^{+}(\sqrt{-3})\right)$ in the obvious manner, we regard the 3-group $C\left(\mathbf{K}_{19}^{+}(\sqrt{-3})\right)$ as a module over the group ring $\mathbf{Z}[\Gamma]$. It then follows from $|\Gamma|=3$ that any $\mathbf{Z}[\Gamma]$-submodule of $C\left(\mathbf{K}_{19}^{+}(\sqrt{-3})\right)$ other than $\{1\}$ contains a non-trivial element fixed by $\Gamma$. Hence, by $h\left(\mathbf{K}_{19}^{+}(\sqrt{-3})\right)=9$, the order $\left|C\left(\mathbf{K}_{19}^{+}(\sqrt{-3})\right)^{1-\gamma}\right|$ equals 3 or 1 , so that

$$
C\left(\mathbf{K}_{19}^{+}(\sqrt{-3})\right)^{(1-\gamma)^{2}}=\{1\} .
$$

Let $l$ be the natural map from $C\left(\mathbf{K}_{19,3}(\sqrt{-3})\right)=C^{-}\left(\mathbf{K}_{19,3}(\sqrt{-3})\right)$ to $C\left(\mathbf{K}_{19}^{+}(\sqrt{-3})\right)$ and $N$ the norm map from $C\left(\mathbf{K}_{19}^{+}(\sqrt{-3})\right)$ to $C\left(\mathbf{K}_{19,3}(\sqrt{-3})\right)$. As the prime ideals of $\mathbf{K}_{19,3}(\sqrt{-3})$ dividing 19 are ramified in $\mathbf{K}_{19}^{+}(\sqrt{-3})$, Proposition 1 of [8] implies that $l$ is injective and class field theory shows that $N$ is surjective. However, by (4),

$$
l(N(c))=c^{1+\gamma+\gamma^{2}}=c^{3+\gamma^{2}(1-\gamma)^{2}}=c^{3} \quad \text { for any } c \in C\left(\mathbf{K}_{19}^{+}(\sqrt{-3})\right) .
$$

Therefore we find from $h\left(\mathbf{K}_{19,3}(\sqrt{-3})\right)=3$ that

$$
C^{-}\left(\mathbf{K}_{19}^{+}(\sqrt{-3})\right)=C\left(\mathbf{K}_{19}^{+}(\sqrt{-3})\right) \cong \mathbf{Z} / 9 \mathbf{Z} \text {. }
$$

Since $\mathbf{K}_{57}$ is a quadratic extension over $\mathbf{K}_{19}^{+}(\sqrt{-3})$ with (relative) class number 9 , we then have

$$
C\left(\mathbf{K}_{57}\right)=C^{-}\left(\mathbf{K}_{57}\right) \cong \mathbf{Z} / 9 \mathbf{Z},
$$

which is a result of [15] (for a general argument, cf. Lemma 4 or 6 of [15]). Note that one conversely obtains (5) from this result without difficulty. It is also easy to see that

$$
C^{-}\left(\mathbf{K}_{60,4}\right)=C\left(\mathbf{K}_{60,4}\right) \cong(\mathbf{Z} / 2 \mathbf{Z})^{2} .
$$


In fact, $h^{-}\left(\mathbf{K}_{60,4}\right)=4, \mathbf{K}_{5}(\sqrt{-1}, \sqrt{-3})$ is the genus field of $\mathbf{K}_{60,4}$, and

$$
\operatorname{Gal}\left(\mathbf{K}_{5}(\sqrt{-1}, \sqrt{-3}) / \mathbf{K}_{60,4}\right) \cong(\mathbf{Z} / 2 \mathbf{Z})^{2} .
$$

Let $H$ be the Hilbert class field over $\mathbf{Q}(\sqrt{-1}, \sqrt{17})$ so that $H$ is a Galois extension over $\mathbf{Q}$. By $h(\mathbf{Q}(\sqrt{-1}, \sqrt{17}))=2, H$ is a quartic abelian extension over $\mathbf{Q}(\sqrt{17})$. As the prime ideals of $\mathbf{Q}(\sqrt{-1}, \sqrt{17})$ dividing 2 are ramified for $\mathbf{Q}(\sqrt{-1}, \sqrt{17}) / \mathbf{Q}(\sqrt{17})$ and unramified in $H, H$ must not be cyclic over $\mathbf{Q}(\sqrt{17})$, i.e., $\operatorname{Gal}(H / \mathbf{Q}(\sqrt{17})) \cong(\mathbf{Z} / 2 \mathbf{Z})^{2}$. On the other hand, the composite $H \mathbf{K}_{17,4}$ is a quadratic extension over $\mathbf{K}_{17,4}(\sqrt{-1})$, since the prime ideals of $\mathbf{Q}(\sqrt{-1}, \sqrt{17})$ dividing 17 are ramified in $H \mathbf{K}_{17,4}$. Now, $\mathbf{K}_{68,4}$ is a quadratic extension over $\mathbf{Q}(\sqrt{17})$ and, by genus theory, $\mathbf{K}_{17,4}(\sqrt{-1})$ is an unramified quadratic extension over $\mathbf{K}_{68,4}$. We then see from $H \mathbf{K}_{17,4}=H \mathbf{K}_{68,4}$ that $H \mathbf{K}_{17,4}$ is an unramified quartic abelian extension over $\mathbf{K}_{68,4}$. However,

$$
\operatorname{Gal}\left(H \mathbf{K}_{17,4} / \mathbf{K}_{68,4}\right) \cong \operatorname{Gal}\left(H / H \cap \mathbf{K}_{68,4}\right) \subseteq \operatorname{Gal}(H / \mathbf{Q}(\sqrt{17})) \cong(\mathbf{Z} / 2 \mathbf{Z})^{2} .
$$

Thus $\operatorname{Gal}\left(H \mathbf{K}_{17,4} / \mathbf{K}_{68,4}\right) \cong(\mathbf{Z} / 2 \mathbf{Z})^{2}$. Since $h^{-}\left(\mathbf{K}_{68,4}\right)=4$, it follows that

$$
C^{-}\left(\mathbf{K}_{68,4}\right)=C\left(\mathbf{K}_{68,4}\right) \cong(\mathbf{Z} / 2 \mathbf{Z})^{2} \text {. }
$$

We can similarly obtain, by $h^{-}\left(\mathbf{K}_{68,8}\right)=4$,

$$
C^{-}\left(\mathbf{K}_{68,8}\right)=C\left(\mathbf{K}_{68,8}\right) \cong(\mathbf{Z} / 2 \mathbf{Z})^{2} \text {, }
$$

starting with the fact that $H \mathbf{K}_{17,4}$ is an abelian quartic extension over $\mathbf{K}_{17,4}$. Note that $H=\mathbf{Q}(\sqrt{17}, \sqrt{1+4 \sqrt{-1}})$, so that $\mathbf{K}_{68,4}(\sqrt{1+4 \sqrt{-1}})$ and $\mathbf{K}_{68,8}(\sqrt{1+4 \sqrt{-1}})$ are the Hilbert class fields over $\mathbf{K}_{68,4}$ and $\mathbf{K}_{68,8}$, respectively. It is easy to see that $C(\mathbf{Q}(\sqrt{-17})) \cong \mathbf{Z} / 4 \mathrm{Z}$. In virtue of the well-known result

$$
C\left(\mathbf{K}_{68}\right)=C^{-}\left(\mathbf{K}_{68}\right) \cong \mathbf{Z} / 8 \mathbf{Z}
$$

(cf. [3]), we still easily see that

$$
\begin{aligned}
C\left(\mathbf{K}_{17,4}(\sqrt{-1})\right) & =C^{-}\left(\mathbf{K}_{17,4}(\sqrt{-1})\right) \cong C^{-}\left(\mathbf{K}_{17,8}(\sqrt{-1})\right) \\
& =C\left(\mathbf{K}_{17,8}(\sqrt{-1})\right) \cong \mathbf{Z} / 8 \mathbf{Z},
\end{aligned}
$$

because $h^{-}\left(\mathbf{K}_{17,8}(\sqrt{-1})\right)=h^{-}\left(\mathbf{K}_{17,4}(\sqrt{-1})\right)=8$ and the prime ideals of $\mathbf{K}_{17,4}(\sqrt{-1})$ dividing 17 are totally ramified in $\mathbf{K}_{68}$.

As already shown in [6],

$$
C\left(\mathbf{K}_{77}\right)=C^{-}\left(\mathbf{K}_{77}\right) \cong(\mathbf{Z} / 4 \mathbf{Z})^{4} \oplus(\mathbf{Z} / 5 \mathbf{Z}) .
$$

Furthermore,

$$
C^{-}\left(\mathbf{K}_{7} \mathbf{K}_{11}^{+}\right)=C\left(\mathbf{K}_{7} \mathbf{K}_{11}^{+}\right) \cong(\mathbf{Z} / 2 \mathbf{Z})^{4} \oplus(\mathbf{Z} / 5 \mathbf{Z}) ;
$$

in fact, $h^{-}\left(\mathbf{K}_{7} \mathbf{K}_{11}^{+}\right)=2^{4} \cdot 5, h\left(\mathbf{K}_{7}\right)=1$, and so Theorem 2.14 of [12] implies that $r_{2}\left(\mathbf{K}_{7} \mathbf{K}_{11}^{+}\right)$is divisible by 4 , the order of 2 modulo $5=\left[\mathbf{K}_{7} \mathbf{K}_{11}^{+}: \mathbf{K}_{7}\right]$. We can also see that $h^{-}\left(\mathbf{K}_{7}^{+} \mathbf{K}_{11}\right)=2^{4}, h\left(\mathbf{K}_{7}^{+}(\sqrt{-11})\right)=1,\left[\mathbf{K}_{7}^{+} \mathbf{K}_{11}: \mathbf{K}_{7}^{+}(\sqrt{-11})\right]=5$, and hence $4 \mid r_{2}\left(\mathbf{K}_{7}^{+} \mathbf{K}_{11}\right)$. Therefore

$$
C^{-}\left(\mathbf{K}_{7}^{+} \mathbf{K}_{11}\right)=C\left(\mathbf{K}_{7}^{+} \mathbf{K}_{11}\right) \cong(\mathbf{Z} / 2 \mathbf{Z})^{4} .
$$


Since

$$
\begin{gathered}
C(\mathbf{Q}(\sqrt{-21})) \cong(\mathbf{Z} / 2 \mathbf{Z})^{2}, \quad h^{-}\left(\mathbf{K}_{7}^{+}(\sqrt{-21})\right)=4, \\
{\left[\mathbf{K}_{7}^{+}(\sqrt{-21}): \mathbf{Q}(\sqrt{-21})\right]=3,}
\end{gathered}
$$

we obtain

$$
C\left(\mathbf{K}_{7}^{+}(\sqrt{-21})\right)=C^{-}\left(\mathbf{K}_{7}^{+}(\sqrt{-21})\right) \cong(\mathbf{Z} / 2 \mathbf{Z})^{2} .
$$

It is shown in [6] that

$$
C\left(\mathbf{K}_{87}\right)=C^{-}\left(\mathbf{K}_{87}\right) \cong(\mathbf{Z} / 8 \mathbf{Z})^{3} \oplus(\mathbf{Z} / 3 \mathbf{Z}) .
$$

In addition to this, the norm map $C\left(\mathbf{K}_{87}\right) \rightarrow C\left(\mathbf{K}_{29}^{+}(\sqrt{-3})\right)$ is surjective since the prime ideal of $\mathbf{K}_{29}^{+}(\sqrt{-3})$ dividing 29 is ramified in $\mathbf{K}_{87}$. On the other hand, $h(\mathbf{Q}(\sqrt{-3}, \sqrt{29}))=3,\left[\mathbf{K}_{29}^{+}(\sqrt{-3}): \mathbf{Q}(\sqrt{-3}, \sqrt{29})\right]=7$, and hence, by Theorem 2.14 of [12], 3 divides both $r_{2}\left(\mathbf{K}_{29}^{+}(\sqrt{-3})\right)$ and the 2-rank of the factor group $C\left(\mathbf{K}_{29}^{+}(\sqrt{-3})\right) /\left\{c^{2} \mid c \in C\left(\mathbf{K}_{29}^{+}(\sqrt{-3})\right)\right\}$. Hence we have

$$
C^{-}\left(\mathbf{K}_{29}^{+}(\sqrt{-3})\right)=C\left(\mathbf{K}_{29}^{+}(\sqrt{-3})\right) \cong(\mathbf{Z} / 4 \mathbf{Z})^{3} \oplus(\mathbf{Z} / 3 \mathbf{Z})
$$

by $h^{-}\left(\mathbf{K}_{29}^{+}(\sqrt{-3})\right)=2^{6} \cdot 3$. As

$$
h^{-}\left(\mathbf{K}_{29,7}(\sqrt{-3})\right)=2^{3}, \quad h(\mathbf{Q}(\sqrt{-3}))=1, \quad\left[\mathbf{K}_{29,7}(\sqrt{-3}): \mathbf{Q}(\sqrt{-3})\right]=7,
$$

we obtain, again from Theorem 2.14 of [12],

$$
C^{-}\left(\mathbf{K}_{29,7}(\sqrt{-3})\right)=C\left(\mathbf{K}_{29,7}(\sqrt{-3})\right) \cong(\mathbf{Z} / 2 \mathbf{Z})^{3} .
$$

Let $H^{\prime}$ be the Hilbert class field over $\mathbf{K}_{29,7}(\sqrt{-3})$. Then $H^{\prime}$ is a Galois extension over $\mathbf{K}_{29,7}$ and, by the above, $\operatorname{Gal}\left(H^{\prime} / \mathbf{K}_{29,7}(\sqrt{-3})\right) \cong(\mathbf{Z} / 2 \mathbf{Z})^{3}$. Let $T$ be the inertia group for $H^{\prime} / \mathbf{K}_{29,7}$ of the prime ideal of $\mathbf{K}_{29,7}$ dividing 3. Clearly, $|T|=2$ and $\operatorname{Gal}\left(H^{\prime} / \mathbf{K}_{29,7}\right)$ is a semidirect product of $\operatorname{Gal}\left(H^{\prime} / \mathbf{K}_{29,7}(\sqrt{-3})\right)$ by $T$. The group ring $\mathbf{Z}\left[\mathrm{Gal}\left(\mathbf{K}_{29,7}(\sqrt{-3}) / \mathbf{K}_{29,7}\right)\right]$ acts on $C\left(\mathbf{K}_{29,7}(\sqrt{-3})\right)$ in the obvious manner. Since $h\left(\mathbf{K}_{29,7}\right)=1$, we have $C\left(\mathbf{K}_{29,7}(\sqrt{-3})\right)^{1+\tau}=\{1\}$, where $\tau$ is the non-trivial element of $\operatorname{Gal}\left(\mathbf{K}_{29,7}(\sqrt{-3}) / \mathbf{K}_{29,7}\right)$. Thus

$$
c^{\tau}=c^{-1}=c \quad \text { for any } c \in C\left(\mathbf{K}_{29,7}(\sqrt{-3})\right) .
$$

Therefore, by class field theory, $H^{\prime}$ is abelian over $\mathbf{K}_{29,7}$ so that

$$
\operatorname{Gal}\left(H^{\prime} / \mathbf{K}_{29,7}\right)=T \times \operatorname{Gal}\left(H^{\prime} / \mathbf{K}_{29,7}(\sqrt{-3})\right) \cong(\mathbf{Z} / 2 \mathbf{Z})^{4} .
$$

Furthermore, the prime ideal of $\mathbf{K}_{29,7}(\sqrt{-3})$ dividing 29 is ramified in $\mathbf{K}_{29}^{+}(\sqrt{-3})$ and, by genus theory, $\mathbf{K}_{29}^{+}(\sqrt{-3})$ is an unramified quadratic extension over $\mathbf{K}_{29,7}(\sqrt{-87})$. Hence $H^{\prime} \mathbf{K}_{29}^{+}$is an unramified abelian extension over $\mathbf{K}_{29,7}(\sqrt{-87})$ of degree $2^{4}$. On the other hand, from $H^{\prime} \mathbf{K}_{29}^{+}=H^{\prime} \mathbf{K}_{29,7}(\sqrt{-87})$ it follows that

$$
\operatorname{Gal}\left(H^{\prime} \mathbf{K}_{29}^{+} / \mathbf{K}_{29,7}(\sqrt{-87})\right) \cong \operatorname{Gal}\left(H^{\prime} / H^{\prime} \cap \mathbf{K}_{29,7}(\sqrt{-87})\right) \subseteq \operatorname{Gal}\left(H^{\prime} / \mathbf{K}_{29,7}\right) \text {. }
$$

We therefore have

$$
\operatorname{Gal}\left(H^{\prime} \mathbf{K}_{29}^{+} / \mathbf{K}_{29,7}(\sqrt{-87})\right) \cong(\mathbf{Z} / 2 \mathbf{Z})^{4} .
$$

However, $h^{-}\left(\mathbf{K}_{29,7}(\sqrt{-87})\right)=2^{4} \cdot 3$. Hence

$$
C^{-}\left(\mathbf{K}_{29,7}(\sqrt{-87})\right)=C\left(\mathbf{K}_{29,7}(\sqrt{-87})\right) \cong(\mathbf{Z} / 2 \mathbf{Z})^{4} \oplus(\mathbf{Z} / 3 \mathbf{Z}) .
$$


Let $\theta=1+3 /\left(\zeta_{7}+\zeta_{7}^{-1}\right)$, so that

$$
\theta \in \mathbf{K}_{7}^{+}, \quad \theta^{3}+3 \theta^{2}-18 \theta-13=0, \quad \theta^{3} \equiv(\theta+1)^{2} \quad(\bmod 4) .
$$

Let $\rho$ be a non-trivial element of $\operatorname{Gal}\left(\mathbf{K}_{7}^{+} / \mathbf{Q}\right)$. As the prime 13 is decomposed in $\mathbf{K}_{7}^{+}$, the principal ideals $(\theta),\left(\theta^{\rho}\right),\left(\theta^{\rho^{2}}\right)$ are the distinct prime ideals of $\mathbf{K}_{7}^{+}$dividing 13. These prime ideals are ramified in $\mathbf{K}_{7}^{+}(\sqrt{-91})$, and $\mathbf{K}_{7}(\sqrt{13})$ is the genus field of $\mathbf{K}_{7}^{+}(\sqrt{-91})$. Hence, as in the case of $\mathbf{L}_{65,12}$, we can see from (6) that $\mathbf{K}_{7}\left(\sqrt{13}, \sqrt{\theta}, \sqrt{\theta^{\rho}}\right)$ is an unramified abelian extension over $\mathbf{K}_{7}^{+}(\sqrt{-91})$ whose Galois group over $\mathbf{K}_{7}^{+}(\sqrt{-91})$ is isomorphic to $(\mathbf{Z} / 2 \mathbf{Z})^{3}$. However, $h^{-}\left(\mathbf{K}_{7}^{+}(\sqrt{-91})\right)=2^{3}$. Thus we have

$$
C^{-}\left(\mathbf{K}_{7}^{+}(\sqrt{-91})\right)=C\left(\mathbf{K}_{7}^{+}(\sqrt{-91})\right) \cong(\mathbf{Z} / 2 \mathbf{Z})^{3} .
$$

Since $h^{-}\left(\mathbf{K}_{7}^{+} \mathbf{K}_{13,3}(\sqrt{-91})\right)=2^{3} \cdot 7$ and $\left[\mathbf{K}_{7}^{+} \mathbf{K}_{13,3}(\sqrt{-91}): \mathbf{K}_{7}^{+}(\sqrt{-91})\right]=3$, we also have

$$
C\left(\mathbf{K}_{7}^{+} \mathbf{K}_{13,3}(\sqrt{-91})\right)=C^{-}\left(\mathbf{K}_{7}^{+} \mathbf{K}_{13,3}(\sqrt{-91})\right) \cong(\mathbf{Z} / 2 \mathbf{Z})^{3} \oplus(\mathbf{Z} / 7 \mathbf{Z}) .
$$

Now, $h(\mathbf{Q}(\sqrt{-7}, \sqrt{13}))=1, h^{-}\left(\mathbf{K}_{7}(\sqrt{13})\right)=2^{2},\left[\mathbf{K}_{7}(\sqrt{13}): \mathbf{Q}(\sqrt{-7}, \sqrt{13})\right]$ $=3, h\left(\mathbf{K}_{13,4}\right)=1, h^{-}\left(\mathbf{K}_{7}^{+} \mathbf{K}_{13,4}\right)=2^{2}$, and $\left[\mathbf{K}_{7}^{+} \mathbf{K}_{13,4}: \mathbf{K}_{13,4}\right]=3$. It therefore follows from Theorem 2.14 of [12] that

$$
C^{-}\left(\mathbf{K}_{7}(\sqrt{13})\right)=C\left(\mathbf{K}_{7}(\sqrt{13})\right) \cong(\mathbf{Z} / 2 \mathbf{Z})^{2} \cong C\left(\mathbf{K}_{7}^{+} \mathbf{K}_{13,4}\right)=C^{-}\left(\mathbf{K}_{7}^{+} \mathbf{K}_{13,4}\right) .
$$

Furthermore, $h^{-}\left(\mathbf{K}_{7} \mathbf{K}_{13}^{+}\right)=2^{2} \cdot 7 \cdot 13, h^{-}\left(\mathbf{K}_{7}^{+} \mathbf{K}_{13}\right)=2^{2} \cdot 37$, and

$$
\left[\mathbf{K}_{7} \mathbf{K}_{13}^{+}: \mathbf{K}_{7}(\sqrt{13})\right]=\left[\mathbf{K}_{7}^{+} \mathbf{K}_{13}: \mathbf{K}_{7}^{+} \mathbf{K}_{13,4}\right]=3 \text {. }
$$

Hence

$$
\begin{gathered}
C\left(\mathbf{K}_{7} \mathbf{K}_{13}^{+}\right)=C^{-}\left(\mathbf{K}_{7} \mathbf{K}_{13}^{+}\right) \cong(\mathbf{Z} / 2 \mathbf{Z})^{2} \oplus(\mathbf{Z} / 7 \mathbf{Z}) \oplus(\mathbf{Z} / 13 \mathbf{Z}), \\
C\left(\mathbf{K}_{7}^{+} \mathbf{K}_{13}\right)=C^{-}\left(\mathbf{K}_{7}^{+} \mathbf{K}_{13}\right) \cong(\mathbf{Z} / 2 \mathbf{Z})^{2} \oplus(\mathbf{Z} / 37 \mathbf{Z}) .
\end{gathered}
$$

It is known (cf. [6]) that

$$
C\left(\mathbf{K}_{91}\right)=C^{-}\left(\mathbf{K}_{91}\right) \cong(\mathbf{Z} / 4 \mathbf{Z})^{2} \oplus(\mathbf{Z} / 7 \mathbf{Z}) \oplus(\mathbf{Z} / 13 \mathbf{Z}) \oplus(\mathbf{Z} / 37 \mathbf{Z}) .
$$

This fact imples

$$
C\left(\mathbf{K}_{7} \mathbf{K}_{13,4}\right)=C^{-}\left(\mathbf{K}_{7} \mathbf{K}_{13,4}\right) \cong(\mathbf{Z} / 4 \mathrm{Z})^{2}
$$

since $h^{-}\left(\mathbf{K}_{7} \mathbf{K}_{13,4}\right)=2^{4}$ and $\left[K_{91}: K_{7} \mathbf{K}_{13,4}\right]=3$. As asserted in [15],

$$
C\left(\mathbf{K}_{\mathbf{9 3}}\right)=C^{-}\left(\mathbf{K}_{\mathbf{9 3}}\right) \cong(\mathbf{Z} / \mathbf{9 Z}) \oplus(\mathbf{Z} / 5 \mathbf{Z}) \oplus(\mathbf{Z} / 151 \mathbf{Z}) \text {. }
$$

Hence

$$
C^{-}\left(\mathbf{K}_{31,6}(\sqrt{-3})\right) \cong \mathbf{Z} / 9 \mathbf{Z}
$$

by $h^{-}\left(\mathbf{K}_{31,6}(\sqrt{-3})\right)=3^{2}$ and $\left[\mathbf{K}_{93}: \mathbf{K}_{31,6}(\sqrt{-3})\right]=5$.

We next obtain

$$
C^{-}\left(\mathbf{K}_{19,3}(\sqrt{-95})\right) \cong \mathbf{Z} / 8 \mathbf{Z}, \quad C^{-}\left(\mathbf{K}_{19}^{+}(\sqrt{-95})\right) \cong(\mathbf{Z} / 8 \mathbf{Z}) \oplus(\mathbf{Z} / 19 \mathbf{Z})
$$

from $C(\mathbf{Q}(\sqrt{-95})) \cong \mathbf{Z} / 8 \mathbf{Z}$. Indeed,

$$
h^{-}\left(\mathbf{K}_{19,3}(\sqrt{-95})\right)=8, \quad h^{-}\left(\mathbf{K}_{19}^{+}(\sqrt{-95})\right)=8 \cdot 19,
$$

$\left[\mathbf{K}_{19,3}(\sqrt{-95}): \mathbf{Q}(\sqrt{-95})\right]=\left[\mathbf{K}_{19}^{+}(\sqrt{-95}): \mathbf{K}_{19,3}(\sqrt{-95})\right]=3$. 
Since $\mathbf{Q}(\sqrt{5}, \sqrt{-19})$ is the genus field of $\mathbf{Q}(\sqrt{-95})$, we also obtain from $h^{-}(\mathbf{Q}(\sqrt{5}, \sqrt{-19}))=4$ that

$$
C^{-}(\mathbf{Q}(\sqrt{5}, \sqrt{-19}))=C(\mathbf{Q}(\sqrt{5}, \sqrt{-19})) \cong \mathbf{Z} / 4 \mathbf{Z}
$$

Furthermore, $h^{-}\left(\mathbf{K}_{19,6}(\sqrt{5})\right)=4, h^{-}\left(\mathbf{K}_{19}(\sqrt{5})\right)=4 \cdot 19, h^{-}\left(\mathbf{K}_{5}(\sqrt{-19})\right) \quad(=$ $h\left(\mathbf{K}_{5}(\sqrt{-19})\right)=4, h^{-}\left(\mathbf{K}_{5} \mathbf{K}_{19,6}\right)=4 \cdot 13, h^{-}\left(\mathbf{K}_{95}\right)=4 \cdot 13 \cdot 19 \cdot 109$, $\mathbf{K}_{19,6}(\sqrt{5})$ is a cubic extension over $\mathbf{Q}(\sqrt{5}, \sqrt{-19}), \mathbf{K}_{19}(\sqrt{5})$ is a cubic extension over $\mathbf{K}_{19,6}(\sqrt{5})$, the prime ideals of $\mathbf{Q}(\sqrt{5}, \sqrt{-19})$ dividing 5 are ramified in $\mathbf{K}_{5}(\sqrt{-19}), \mathbf{K}_{5} \mathbf{K}_{19,6}$ is a cubic extension over $\mathbf{K}_{5}(\sqrt{-19})$, and $\mathbf{K}_{95}$ is a cubic extension over $\mathbf{K}_{5} \mathbf{K}_{19,6}$. Thus $C^{-}\left(\mathbf{K}_{19,6}(\sqrt{5})\right), C^{-}\left(\mathbf{K}_{19}(\sqrt{5})\right)$, $C^{-}\left(\mathbf{K}_{5}(\sqrt{-19})\right) \quad\left(=C\left(\mathbf{K}_{5}(\sqrt{-19})\right)\right), C^{-}\left(\mathbf{K}_{5} \mathbf{K}_{19,6}\right)$, and $C^{-}\left(\mathbf{K}_{95}\right)$ are cyclic groups. It is known that

$$
C\left(\mathbf{K}_{\mathbf{9 6}}\right)=C^{-}\left(\mathbf{K}_{\mathbf{9 6}}\right) \cong(\mathbf{Z} / 3 \mathbf{Z})^{2}
$$

(cf. [15]), while $h^{-}\left(\mathbf{K}_{32}^{+}(\sqrt{-3})\right)=3^{2}$ and $h^{-}\left(\mathbf{K}_{96,8}(\sqrt{-1})\right)=h^{-}\left(\mathbf{K}_{96,8}(\sqrt{3})\right)=$ $h^{-}\left(\mathbf{K}_{96,8}\right)=2 \cdot 3^{2}$. Hence, noting $\left[\mathbf{K}_{96}: \mathbf{Q}\right]=2^{5}$, we easily have

$$
\begin{gathered}
C\left(\mathbf{K}_{32}^{+}(\sqrt{-3})\right)=C^{-}\left(\mathbf{K}_{32}^{+}(\sqrt{-3})\right) \cong(\mathbf{Z} / 3 \mathbf{Z})^{2} \\
C\left(\mathbf{K}_{96,8}(\sqrt{-1})\right)=C^{-}\left(\mathbf{K}_{96,8}(\sqrt{-1})\right) \cong C\left(\mathbf{K}_{96,8}(\sqrt{3})\right)=C^{-}\left(\mathbf{K}_{96,8}(\sqrt{3})\right) \\
\cong C\left(\mathbf{K}_{96,8}\right)=C^{-}\left(\mathbf{K}_{96,8} \cong(\mathbf{Z} / 2 \mathbf{Z}) \oplus(\mathbf{Z} / 3 \mathbf{Z})^{2} .\right.
\end{gathered}
$$

Since it is known that

$$
C\left(\mathbf{K}_{99}\right)=C^{-}\left(\mathbf{K}_{99}\right) \cong(\mathbf{Z} / 3 \mathbf{Z}) \oplus(\mathbf{Z} / 31 \mathbf{Z})^{2}
$$

(cf. [15]), the assertion II is now completely proved.

4

We conclude the paper by verifying the result stated at the end of the introduction. Let $k$ be any imaginary abelian field in $\mathscr{H}$. Let $\mathscr{R}$ denote the subset of $\mathscr{H}$ defined by

$$
\mathscr{R}=\left\{\mathbf{K}_{71}, \mathbf{K}_{73}, \mathbf{K}_{73,24}, \mathbf{K}_{79}, \mathbf{K}_{79,26}, \mathbf{K}_{83}, \mathbf{K}_{89}, \mathbf{K}_{97}, \mathbf{K}_{97,32}\right\}
$$

so that $\mathscr{H} \backslash \mathscr{R}$ is the family of imaginary abelian fields $k^{\prime}$ in $\mathscr{H}$ for which either $\left[k^{\prime}: \mathbf{Q}\right] \leq 23$ or $f\left(k^{\prime}\right)$ is not a prime number $\geq 71$. We then have:

III. Under the assumption $k \in \mathscr{H} \backslash \mathscr{R}, C(k)$ is cyclic if and only if $C^{-}(k)$ is cyclic.

Proof. Let

$$
\begin{gathered}
\mathbf{L}_{80,4}=\mathbf{Q}(\sqrt{-10-3 \sqrt{10}}), \quad \mathbf{L}_{80,4}^{\prime}=\mathbf{Q}(\sqrt{-10-\sqrt{10}}), \\
\mathbf{L}_{85,4}=\mathbf{Q}(\sqrt{-85-6 \sqrt{85}}), \quad \mathbf{L}_{85,4}^{\prime}=\mathbf{Q}(\sqrt{-85-2 \sqrt{85}}), \\
\mathbf{L}_{85,8}=\mathbf{Q}\left(\left(\zeta_{5}-\zeta_{5}^{-1}\right)\left(\zeta_{17}-\zeta_{17}^{-1}\right)\left(\zeta_{17}^{4}-\zeta_{17}^{-4}\right)-\left(\zeta_{5}^{2}-\zeta_{5}^{-2}\right)\left(\zeta_{17}^{2}-\zeta_{17}^{-2}\right)\left(\zeta_{17}^{8}-\zeta_{17}^{-8}\right)\right), \\
\mathbf{L}_{85,8}^{\prime}=\mathbf{Q}\left(\left(\zeta_{5}-\zeta_{5}^{-1}\right)\left(\zeta_{17}-\zeta_{17}^{-1}\right)\left(\zeta_{17}^{4}-\zeta_{17}^{-4}\right)+\left(\zeta_{5}^{2}-\zeta_{5}^{-2}\right)\left(\zeta_{17}^{2}-\zeta_{17}^{-2}\right)\left(\zeta_{17}^{8}-\zeta_{17}^{-8}\right)\right) .
\end{gathered}
$$


These are distinct imaginary cyclic fields such that

$$
\begin{gathered}
f\left(\mathbf{L}_{80,4}\right)=f\left(\mathbf{L}_{80,4}^{\prime}\right)=80 \\
f\left(\mathbf{L}_{85,4}\right)=f\left(\mathbf{L}_{85,4}^{\prime}\right)=f\left(\mathbf{L}_{85,8}\right)=f\left(\mathbf{L}_{85,8}^{\prime}\right)=85, \\
{\left[\mathbf{L}_{80,4}: \mathbf{Q}\right]=\left[\mathbf{L}_{80,4}^{\prime}: \mathbf{Q}\right]=\left[\mathbf{L}_{85,4}: \mathbf{Q}\right]=\left[\mathbf{L}_{85,4}^{\prime}: \mathbf{Q}\right]=4,} \\
{\left[\mathbf{L}_{85,8}: \mathbf{Q}\right]=\left[\mathbf{L}_{85,8}^{\prime}: \mathbf{Q}\right]=8 .}
\end{gathered}
$$

Let

$$
\mathscr{N}=\left\{\begin{array}{l}
\mathbf{L}_{80,4}(\sqrt{-1}), \mathbf{L}_{80,4}^{\prime}(\sqrt{-1}), \mathbf{L}_{80,4}(\sqrt{-5}), \mathbf{L}_{80,4}^{\prime}(\sqrt{-5}), \\
\mathbf{L}_{80,4}, \quad \mathbf{L}_{80,4}^{\prime}, \quad \mathbf{L}_{85,8}, \quad \mathbf{L}_{85,8}^{\prime}, \mathbf{L}_{85,4}, \mathbf{L}_{85,4}^{\prime}
\end{array}\right\},
$$

so that $\mathscr{N} \subseteq \mathscr{H} \backslash \mathscr{R}$. Assume now that $h^{-}(k)$ is square-free and that $k$ lies in $\mathscr{H} \backslash \mathscr{R}$. Then the results in $[1,4,11,12]$ imply that $h(k)$ is not square-free if and only if $k$ lies in $\mathscr{N}$, and that

$$
\begin{gathered}
h\left(\mathbf{L}_{80,4}(\sqrt{-1})\right)=h\left(\mathbf{L}_{80,4}(\sqrt{-5})\right)=h\left(\mathbf{L}_{80,4}\right)=h\left(\mathbf{L}_{85,8}\right)=h\left(\mathbf{L}_{85,4}\right)=4, \\
h\left(\mathbf{L}_{80,4}^{\prime}(\sqrt{-1})\right)=h\left(\mathbf{L}_{80,4}^{\prime}(\sqrt{-5})\right)=h\left(\mathbf{L}_{80,4}^{\prime}\right)=h\left(\mathbf{L}_{85,4}^{\prime}\right)=4 \cdot 5, \\
h\left(\mathbf{L}_{85,8}^{\prime}\right)=4 \cdot 73 .
\end{gathered}
$$

However, for each $k^{\prime} \in \mathcal{N}$, the genus field of $k^{\prime}$ is a quartic cyclic extension over $k^{\prime}$. It therefore follows that $C\left(k^{\prime}\right)$ is cyclic for every $k^{\prime} \in \mathscr{N}$.

On the other hand, if $k$ lies in $\mathscr{H} \backslash \mathscr{R}$ and $h^{-}(k)$ is not square-free, then $h\left(k^{+}\right)=1$, i.e., $C(k)=C^{-}(k)$ (cf. the proof of II). Hence III is proved.

Remark. In virtue of the results of $[1,11,12], h\left(k^{+}\right)$equals the genus number of $k^{+}$whenever $k$ lies in $\mathscr{H} \backslash \mathscr{R}$. Theorem 2 of [11] further implies that, even in the case $k \in \mathscr{R}, h\left(k^{+}\right)$equals 1 , the genus number of $k^{+}$, if the generalized Riemann hypothesis for the Dedekind zeta function of $k^{+}$is assumed to be true.

Although we omit the details, the results of $[13,17,18]$, together with those of $[1,2,8,11,12,15,16]$, enable us to determine the structure of $C\left(k^{\prime \prime}\right)$ or $C^{-}\left(k^{\prime \prime}\right)$ for several imaginary abelian fields $k^{\prime \prime}$ outside $\mathscr{H}$ (cf. $\left.[6,15]\right)$.

\section{ACKNOWLEDGMENT}

This paper contains part of the second author's master's degree thesis at Tsukuba University. She wishes to thank Professor Tatsuo Kimura, her thesis adviser, for his guidance and helpful comments. The authors are grateful at the same time to the referee who offered several suggestions improving the paper in manuscript.

\section{REFERENCES}

1. H. Bauer, Numerische Bestimmung von Klassenzahlen reeller zyklischer Zahlkörper. J. Number Theory 1 (1969), 161-162.

2. G. Cornell and M. Rosen, Group-theoretic constraints on the structure of the class group, J. Number Theory 13 (1981), 1-11.

3. F. Gerth, The ideal class groups of two cyclotomic fields, Proc. Amer. Math. Soc. 78 (1980), 321-322.

4. H. Hasse, Über die Klassenzahl abelscher Zahlkörper, Akademie-Verlag, Berlin, 1952, and Springer-Verlag, Berlin, 1985. 
5. K. Horie, On the exponents of ideal class groups of cyclotomic fields, Proc. Amer. Math. Soc. 119 (1993), 1049-1052.

6. K. Horie and M. Horie, On the 2-class groups of cyclotomic fields whose maximal real subfields have odd class numbers, Proc. Amer. Math. Soc. (to appear).

7. K. Iwasawa, A note on ideal class groups, Nagoya Math. J. 27 (1966), 239-247.

8. Y. Kida, l-extensions of CM-fields and cyclotomic invariants, J. Number Theory 12 (1980), 519-528.

9. E. E. Kummer, Über die Irregularität von Determinanten, Monatsber. Akad. Wiss. Berlin 1853, 194-200; reprinted in his Collected Papers. Vol. I, pp. 539-545.

10. _ Über die Klassenzahl der aus n-ten Einheitswurzeln gebildeten complexen Zahlen, Monatsber. Akad. Wiss. Berlin 1861, 1051-1053; reprinted in his Collected Papers. Vol. I, pp. 883-885.

11. F. J. van der Linden, Class number computations of real abelian number fields, Math. Comp. 39 (1982), 693-707.

12. J. M. Masley, Class numbers of real cyclic number fields with small conductor, Compositio Math. 37 (1978), 297-319.

13. G. Schrutka von Rechtenstamm, Tabelle der Relativ-Klassenzahlen der Kreiskörper, deren $\phi$-Funktion des Wurzelexponenten (Grad) nicht grösser als 256 ist, Abh. Deutsch. Akad. Wiss. Berlin Kl. Math. Phys. 1964, no. 2.

14. W. Sinnott, On the Stickelberger ideal and the circular units of an abelian field, Invent. Math. 62 (1980), 181-234.

15. K. Tateyama, On the ideal class groups of some cyclotomic fields, Proc. Japan Acad. Ser. A 58 (1982), 333-335.

16. L. C. Washington, Introduction to cyclotomic fields, Springer-Verlag, Berlin, 1982.

17. $\mathrm{K}$. Yoshino and $\mathrm{M}$. Hirabayashi, On the relative class number of the imaginary abelian number field. I, Mem. Coll. Liberal Arts Kanazawa Medical Univ. 9 (1981), 5-53.

18. _ On the relative class number of the imaginary abelian number field. II, Mem. Coll. Liberal Arts Kanazawa Medical Univ. 10 (1982), 33-81.

Department of Mathematics, Tokai University, 1117 Kitakaname, Hiratsuka 259-12, JAPAN

Mashiko Highschool, 2382-1 Hanawa, Mashiko-machi, Haga-gun, Tochigi 321-42, Japan 